\title{
Efficiency and Vectorial Optimization
}

\author{
Vasile Postolică \\ Romanian Academy of Scientists/, Vasile Alecsandri University of Bacău \\ Faculty of Sciences / Department of Mathematics and Informatics /, Bacău, Romania.
}

*Corresponding Author: Vasile Postolică, Romanian Academy of Scientists/, Vasile Alecsandri University of Bacău, Faculty of Sciences / Department of Mathematics and Informatics /, Bacău, Romania.

\begin{abstract}
This scientific research report was presented, in the quality of session chair and as an expert speech, at the $5^{\text {th }}$ International Multi-Disciplinary Conference organized by Institute of Research Engineers and Doctors - IRED, USA September 02 - 03, 2017, Zurich, Switzerland. It is devoted to the general Efficiency presented inside the best appropriate environment of the infinite dimensional Ordered Vector Spaces, with its projections in Vectorial Optimization, that is, in Euclidean spaces $R^{n}, n \in N^{*}$ which can be extended, mutatis mutandis, to $R^{\prime}$ for every non -empty set I. Following our refined recent results, we especially use the largest class of the Convex Cones discovered till now in separated Locally Convex Spaces, named by us "Isac's Cones", and ensuring the existence together with important properties for the efficient points under completeness instead of compactness, with pertinent applications.The couched Open problems are generated by the new links between the General Efficiency, the Vector (Strong) Optimization and the Choquet Boundaries, since the Efficiency is strongly related to the General Optimization, the Potential Theory and conversely, with projections in the new recent and future fields of Scientific Research: Theory and Applications of the Generalized Dynamical Systems, Fixed points Theory, the Best Approximation Theory, the study of the Conically Bounded Sets, the study of the Nuclearity for the Topological Vector Spaces, Vector Optimization for multivalued functions and so on.
\end{abstract}

Keywords: Ordered Vector Space, General Efficiency, Multifunction, Pareto Optimality, Locally Convex Space, Isac's (nuclear, supernormal) Cone (set), Choquet Boundary, Best Approximation, Spline function, Multi-Objective Optimization, Strong and Vectorial Optimal Solution.

\section{INTRODUCTION}

Until now, the basis on which was developed our general concept of the Efficiency in the Ordered Linear Spaces and its Applications, supplied by the Ordered Locally Convex Spaces, is represented about the vastness class of the Convex Cones introduced by Professor Isac in 1981, published in 1983, called by us and, officially recognized, as "Isac's Cone" in 2009, after the acceptance of this last agreed denomination by Professor Isac. To Professor Isac is devoted this scientific research work.We think that it is not possible to obtain more than these results concerning the General Efficiency in the world of the separated Locally Convex Spaces.They represent the most development background. All the elements concerning the ordered topological vector spaces used here are in acordance with Nachbin, L., 1965 and Peressini, A., L., 1967. The bibliography of this research work represents a synthesis of all the examined references.

\section{General EFFicienCy In THE ORdeREd Vector SPaCES}

Let $\mathrm{E}$ be any real or complex vector space with the origin element $\theta$, ordered by a convex cone $K$, that is, $K+K \subseteq K$ and $\chi K \subseteq K$ for all $\chi \in R_{+}, K_{1}$ a non - void subset of $K$ and $A$ a non - empty subset of $\mathrm{E}$. The following definition introduces a new concept of the approximate efficiency, which expresses the general efficiency, generalizes the well known notion of Pareto type efficiency in every Euclidean space and not only. 
Definition 1. (Postolică, $V$., 2002, 2008). We say that $a_{0} \in A$ is a $K_{1}$-minimal efficient point of $A$, in notation, $a_{0} \in \operatorname{MIN}\left(A, K, K_{1}\right)$ if it satisfies one of the following equivalent conditions:

(i) $A \cap\left(a_{0}-K-K_{1}\right) \subseteq a_{0}+K+K_{1}$;

(ii) $\left(K+K_{1}\right) \cap\left(a_{0}-A\right) \subseteq-K-K_{1}$;

In a similar manner one defines the $K_{1}$-maximal efficient points by replacing $K+K_{1}$ with $-\left(K+K_{1}\right)$. The set of such as these points will be denoted by $\operatorname{MAX}\left(A, K, K_{1}\right)$. Whenever $K_{1}=\{\theta\}$ we consider $\operatorname{MIN}\left(A, K, K_{1}\right)=\operatorname{MIN}(A, K)$ and $\operatorname{MAX}\left(A, K, K_{1}\right)=\operatorname{MAX}(A, K)$, respectively. All the efficient points of the set $A$ with respect to the convex cone Kfollowing $K_{1}$ are represented by the set eff $\left(A, K, K_{1}\right)=\operatorname{MIN}\left(A, K, K_{1}\right) \cup \operatorname{MAX}\left(A, K, K_{1}\right)$. Consequently, eff $(A, K)=\operatorname{MIN}(A, K) \cup \operatorname{MAX}(A, K)$. It is obvious that

$A \cap\left(a_{0} \mp K\right) \subseteq a_{0} \pm K_{1} \Rightarrow A \bigcap\left(a_{0} \pm K \pm K_{1}\right) \subseteq a_{0} \mp K \mp K_{1} \Rightarrow A \cap\left(a_{0} \mp K_{1}\right) \subseteq a_{0} \pm K$,

which suggests other concepts for the efficiency and its approximate term in the general ordered linear spaces.

Remark 1. We notice that $a_{0} \in \operatorname{eff}\left(A, K, K_{1}\right)$ iff it is a fixed point for at least one of the multifunctions $F_{\mp}: A \rightarrow 2^{A}$ defined by $F_{\mp}(t)=\left\{a \in A: A \cap\left(a \mp K \mp K_{1}\right) \subseteq t \pm K \pm K_{1}\right\}$, that is, $a_{0} \in F_{\mp}\left(a_{0}\right)$, with the corresponding signs. Consequently, for the existence of these general efficient points we can also apply appropriate fixed points theorems concerning the multifunctions (see, for instance, Cardinali, T., Papalini, F., 1994, Park, S.,1992, Patriche, M.,2013, Zhang Cong Jun, 2005 and any other proper scientific papers).

Remark 2. Németh, A.B., 1989, proved that, whenever $K_{1} \subseteq K \backslash\{\theta\}$, the existence of this new type of the efficient points for the lower bounded sets characterizes the semi - Archimedian Ordered Vector Spaces and the Regular Ordered Locally Convex Spaces.

Remark 3. Whenever $K$ is pointed, that is, $K \cap(-K)=\{\theta\}$, then $a_{0} \in \operatorname{MIN}\left(A, K, K_{1}\right)\left(a_{0} \in \operatorname{MAX}\left(A, K, K_{1}\right)\right)$ means that $A \cap\left(a_{0} \mp K \mp K_{1}\right)=\varnothing$ or, equivalently, $\left( \pm K+K_{1}\right) \cap\left(a_{0}-A\right)=\varnothing$ for $\theta \notin K_{1}$ and $A \cap\left(a_{0} \mp K \mp K_{1}\right)=\left\{a_{0}\right\}$, respectively, if $\theta \in K_{1}$. Clearly, $K \subseteq K+K_{1}$ if $\theta \in K_{1}$ and, if the convex cone $K$ is pointed, then $K \subseteq K+K_{1} \Leftrightarrow \theta \in K_{1}$. This inclusion is not always valid as we can see taking $E=R^{2}, K=\left\{(x ; y) \in R^{2}: x \in R, y \geq 0\right\}$ and $K_{1}=\{(1,1)\}$, but, in our considered case, it remains true for $K_{1}=\{(0,-1)\}$. Whenever $K_{1}=\{\theta\}$, from Definition 1 one obtains the usual concept of the efficient (Pareto minimal, optimal or admissible) point : $a_{0} \in M I N(A, K)(M A X(A, K))$ if it fulfils (i), (ii) or any of the next equivalent properties:

(iii) $(\mathrm{A} \pm \mathrm{K}) \cap\left(\mathrm{a}_{0} \mp \mathrm{K}\right) \subseteq \mathrm{a}_{0} \pm \mathrm{K}$;

(iv) $\mathrm{K} \cap\left(\mathrm{a}_{0}-\mathrm{A} \mp \mathrm{K}\right) \subseteq \mp \mathrm{K}$

This shows that $a_{0}$ is a fixed point for at least one of the following multifunctions:

$$
F_{1}: A \rightarrow A, F_{1}(t)=\{a \in A: A \bigcap(a \mp K) \subseteq t \pm K\},
$$




$$
\begin{gathered}
F_{2}: A \rightarrow A, F_{2}(t)=\{a \in A: A \cap(t \mp K) \subseteq a \pm K\}, \\
F_{3}: A \rightarrow A, F_{3}(t)=\{a \in A:(A \pm K) \cap(a \mp K) \subseteq t \pm K\}, \\
F_{4}: A \rightarrow A, F_{4}(t)=\{a \in A:(A \pm K) \cap(t \mp K) \subseteq a \pm K\},
\end{gathered}
$$

that is, $a_{0} \in F_{i}\left(a_{0}\right)$ for some $i=\overline{1,4}$. If, in addition, $K$ is pointed, then $\mathrm{a}_{0} \in \mathrm{A}$ is an efficient point of A with respect to $K$ if and only if one of the following equivalent relations holds :

(v) $\mathrm{A} \cap\left(\mathrm{a}_{0} \mp \mathrm{K}\right)=\left\{\mathrm{a}_{0}\right\}$;

(vi) $A \cap\left(a_{0} \mp K \backslash\{\theta\}\right)=\varnothing$;

(vii) $( \pm K) \cap\left(a_{0}-A\right)=\{\theta\}$;

(viii) $( \pm \mathrm{K} \backslash\{\theta\}) \cap\left(\mathrm{a}_{0}-\mathrm{A}\right)=\varnothing$;

(ix) $(\mathrm{A} \pm \mathrm{K}) \cap\left[\mathrm{a}_{0} \mp(\mathrm{K} \backslash\{\theta\})\right]=\varnothing$.

We also notice that

$\operatorname{MIN}(A, K)(\operatorname{MAX}(A, K))=\bigcap_{\{0\} \neq K_{2} \subseteq K} \operatorname{MIN}\left(A, K, K_{2}\right)\left(\bigcap_{\{0\} \neq K_{2} \subseteq-K} \operatorname{MAX}\left(A, K, K_{2}\right)\right)$. Moreover,

$a_{0} \in e f f(A, K)$ if only if it is a critical (ideal or balance) point ( see, for example, Kim, W. K., Tan, K.K., 2001; Isac,G. 1985; Isac, G., Bulavsky, V. A., Kalashnikov, V., V. 2002; Isac,G., Postolică, V.,1993; Postolică, V., Scarelli, A., Venzi,L., 2001; Postolică, V., 2004 and the corresponding references) for the generalized dynamical systems $\Gamma_{\mp}: A \rightarrow 2^{A}$ defined by $\Gamma_{\mp}(a)=A \cap(a \mp K), a \in A$. In this way, eff $(A, K)$ describes the balance extremum moments for $\Gamma_{\mp}$, which in the abstract market context, expresses the competitive equilibrium/non-equilibrium consisting of the general relation price/consumption. Considering $K_{1}=\{\varepsilon\}(\varepsilon \in K \backslash\{\theta\})$, one obtains that $a_{0} \in e f f\left(A, K, K_{1}\right)$ iff $A \cap\left(a_{0}-\varepsilon \mp K\right)=\varnothing$. In all these cases, for the set $\operatorname{MIN}\left(A, K, K_{1}\right) \quad$ we used the notation $\varepsilon-\operatorname{MIN}(A, K)$. It is obvious that $\operatorname{MIN}(A, K)=\bigcap_{\varepsilon \in K \backslash\{0\}}[\varepsilon-M I N(A, K)]$, with the natural projections for $\operatorname{MAX}(A, K)$ obtained by replacing the convex cone $K$ with $-K$. When referring to the existence of the efficient points, the significant properties of the sets containing these points and the applications in the usual or extended contexts we mention, as a comprehensive bibliography, (Aubin, J.P., 1993; Benson, H. P., 1077, 1979,1983;Borwein, J. M., 1977, 1983;Dauer, J. P., Gallagher, R. J., 1990; Dominiak, Cezary, 2006; Ehrgott, Matthias, 2005;Fishburn, P. C., 1984; Gass, Saul.,I., Harris, Carl., M., 2001; Geoffrion, A. M;, 1968; Hartley, R., 1978; Henig, M. I., 1982; Heyne, Paul, 2000; Hyers, D., H., Isac, G., Rassias,T.M.,1997; Isac, G., 1981, 1983, 1985, 1994, 1998,2003, Isac, G., Bahya, A. O., 2002; Isac, G., Postolică, V.,1989, 1993,1995, 1997, 1999, 2001, 2002, 2009, 2014, 2015, 2016; Isac, G., Tammer,Chr.,2003; Jablonsky, J.,2006; Loridan, P., 1984; Luc, D. T., 1989; Luptáčik, M., Böhm, B., 2006; Németh, A., B., 1989; Ng., K., F., Zheng, X., Y., 2002; Postolică, V.,1989, 1993, 1994, 1997, 1999, 2001, 2002, 2004, 2008, 2009; Songsak Sriboonchitta, Wing - Keung Wong, Sompong Dhopongsa, Hung, T., Nguyen, 2009; Sontag, Z., Zălinescu, C., 2000; Stewart, D., T., 2004; Truong, X. D. H., 1994; Zimmermann, H., 2000), with widely established results.

Open Problem 1. Find general criteria to obtain eff $\left(A, K, K_{1}\right) \subseteq \operatorname{eff}\left(A, \widetilde{K}, K_{1}\right)$ from $K \subseteq \widetilde{K}$ when $K, \widetilde{K}$ are convex cones in $E$ and eff $\left(A, K, K_{1}\right) \subseteq \operatorname{eff}\left(A, K, K_{2}\right)$ if $K_{1} \subseteq K_{2} \subseteq K$, respectively.

In order to offer a first solution, we remark that if $K \subseteq \widetilde{K} \subseteq K+K_{1}$, then 
$\operatorname{eff}\left(A, K, K_{1}\right)=\operatorname{eff}\left(A, \widetilde{K}, K_{1}\right)$ : it is sufficient to prove that $\operatorname{MIN}\left(A, K, K_{1}\right)=\operatorname{MIN}\left(A, \widetilde{K}, K_{1}\right)$. Indeed, if $a_{0} \in \operatorname{MIN}\left(A, K, K_{1}\right)$, then $A \cap\left(a_{0}-K-K_{1}\right) \subseteq a_{0}+K+K_{1}$. On the other hand, $A \cap\left(a_{0}-\widetilde{K}-K_{1}\right) \subseteq A \cap\left(a_{0}-K-K_{1}\right) \subseteq a_{0}+K+K_{1} \subseteq a_{0}+\widetilde{K}+K_{1}$, that is, $a_{0} \in \operatorname{MIN}\left(A, \widetilde{K}, K_{1}\right)$. Let now $A \cap\left(a_{0}-\widetilde{K}-K_{1}\right) \subseteq a_{0}+\widetilde{K}+K_{1}$. Then, $A \cap\left(a_{0}-K-K_{1}\right) \subseteq A \cap\left(a_{0}-\widetilde{K}-K_{1}\right) \subseteq a_{0}+\widetilde{K}+K_{1} \subseteq a_{0}+K+K_{1}$. So, $a_{0} \in \operatorname{MIN}\left(A, K, K_{1}\right)$. Consequently, $\operatorname{MIN}\left(A, K, K_{1}\right)=\operatorname{MIN}\left(A, \widetilde{K}, K_{1}\right)$. This completes the proof. In a similar manner one proves that $\operatorname{MAX}\left(A, K, K_{1}\right)=\operatorname{MAX}\left(A, \widetilde{K}, K_{1}\right)$ under the same hypothesis and we conclude that $\operatorname{eff}\left(A, K, K_{1}\right)=e f f\left(A, \widetilde{K}, K_{1}\right)$. Let now $K_{1} \subseteq K_{2} \subseteq K_{1}+K$ and $a_{0} \in \operatorname{MIN}\left(A, K, K_{1}\right)$, that is, $A \cap\left(a_{0}-K-K_{1}\right) \subseteq a_{0}+K+K_{1}$. Then, $A \cap\left(a_{0}-K-K_{2}\right) \subseteq A \cap\left(a_{0}-K-K_{1}\right) \subseteq a_{0}+K+K_{1} \subseteq a_{0}+K+K_{2}$. So, $a_{0} \in M I N\left(A, K, K_{2}\right)$. At the same time, if $A \cap\left(a_{0}-K-K_{2}\right) \subseteq a_{0}+K+K_{2}$, then $A \cap\left(a_{0}-K-K_{1}\right) \subseteq A \cap\left(a_{0}-K-K_{2}\right) \subseteq a_{0}+K+K_{2} \subseteq a_{0}+K+K_{1}$, that is, $a_{0} \in \operatorname{MIN}\left(A, K, K_{1}\right)$. Therefore, $\operatorname{MIN}\left(A, K, K_{1}\right)=\operatorname{MIN}\left(A, K, K_{2}\right)$. The above assumption on $K, K_{1}$ and $K_{2}$ ensures, in a similar manner, that $\operatorname{MAX}\left(A, K, K_{1}\right)=\operatorname{MAX}\left(A, K, K_{2}\right)$ and thus eff $\left(A, K, K_{1}\right)=\operatorname{eff}\left(A, K, K_{2}\right)$. We also have eff $(A, \widetilde{K}) \subseteq \operatorname{eff}(A, K)$ if $K$ is pointed and $K \subseteq \widetilde{K}$. The hypotheses $K \subseteq \widetilde{K} \subseteq K+K_{1}$ and $K_{1} \subseteq K_{2} \subseteq K_{1}+K$ seem to be too strong to solve the formulated open problem.

Open Problem 2. Let us denote by $2^{\mathrm{E}}, 2^{\mathrm{K}}, 2^{\mathrm{K}_{1}}$ the families of all subsets for $\mathrm{E}$, all the convex cones in $\mathrm{E}$, all the non - empty subsets of these cones, respectively, and the multifunction eff : $2^{\mathrm{E}} \times 2^{\mathrm{K}} \times 2^{\mathrm{K}_{1}} \rightarrow 2^{\mathrm{E}}$ defined by $\left(A, K, K_{1}\right) \in\left(2^{\mathrm{E}} \times 2^{\mathrm{K}} \times 2^{\mathrm{K}_{1}}\right) \rightarrow$ eff $\left(A, K, K_{1}\right) \in 2^{\mathrm{E}}$. The study of the properties for these multifunctions, starting with their restrictions.

Thus, for example, if $\mathrm{E}$ is any linear topological vector space, the lower (upper) semicontinuity and the continuity of this map are important for the stability investigation in the optimization problems concerning the efficiency.

Let us consider the set $\operatorname{SMIN}\left(A, K, K_{1}\right)=\left\{a_{1} \in A: A \subseteq a_{1}+K+K_{1}\right\}$ of all strong minimum points of $A$ with respect to $K$ by $K_{1}$ and $\operatorname{SMAX}\left(A, K, K_{1}\right)=\left\{a_{2} \in A: A \subseteq a_{2}-K-K_{1}\right\}$ the set of all strong maximum points of $A$ with respect to $K$ by $K_{1}$, respectively, and $S\left(A, K, K_{1}\right)=\left\{a_{1} \in A: A \subseteq a_{1}+K+K_{1}\right\} \cup\left\{a_{2} \in A: A \subseteq a_{2}-K-K_{1}\right\}$

The following theorem shows the immediate connection between the approximate efficiency and the strong optimization through the agency of the cone minimal (cone maximal) efficient points.

Theorem 1. If $S\left(A, K, K_{1}\right) \neq \varnothing$, then $S\left(A, K, K_{1}\right)=$ eff $\left(A, K, K_{1}\right)$.

Proof. In fact, we prove that $\operatorname{SMIN}\left(A, K, K_{1}\right) \neq \varnothing \Rightarrow \operatorname{MIN}\left(A, K, K_{1}\right)=\operatorname{SMIN}\left(A, K, K_{1}\right)$ and $\operatorname{SMAX}\left(A, K, K_{1}\right) \neq \varnothing \Rightarrow \operatorname{MAX}\left(A, K, K_{1}\right)=\operatorname{SMAX}\left(A, K, K_{1}\right)$, so it is sufficient to show the first implication. Clearly, $\operatorname{SMIN}\left(A, K, K_{1}\right) \subseteq \operatorname{MIN}\left(A, K, K_{1}\right)$. Indeed, if $a_{0} \in \operatorname{SMIN}\left(A, K, K_{1}\right)$ and $a \in A \cap\left(a_{0}-K-K_{1}\right)$ are arbitrary elements, then $a \in a_{0}+K+K_{1}$, that is, $a_{0} \in \operatorname{MIN}\left(A, K, K_{1}\right)$ by virtue of the point (i) contained in Definition 1 . Suppose now that $\bar{a} \in \operatorname{SMIN}\left(A, K, K_{1}\right) \neq \varnothing$ and 
there exists $a_{0} \in \operatorname{MIN}\left(A, K, K_{1}\right) \backslash \operatorname{SMIN}\left(A, K, K_{1}\right)$. Out of the fact $\bar{a} \in \operatorname{SMIN}\left(A, K, K_{1}\right)$, it follows that $a_{0} \in \bar{a}+K+K_{1}$, that is, $\bar{a} \in a_{0}-K-K_{1}$, from which, since $\bar{a} \in A$ and $a_{0} \in \operatorname{MIN}\left(A, K, K_{1}\right)$, we conclude that $\bar{a} \in a_{0}+K+K_{1}$. Therefore,

$A \subseteq \bar{a}+K+K_{1} \subseteq a_{0}+K+K_{1}$, in contradiction with $a_{0} \notin \operatorname{SMIN}\left(A, K, K_{1}\right)$, as claimed.

Remark 4. It is obvious that we have always $S\left(A, K, K_{1}\right) \subseteq$ eff $\left(A, K, K_{1}\right)$. The previous theorem shows that, every time when there exists at least one strong cone minimum or cone maximum point, the set of all efficient points coincides with the set of all these points, respectively. This is important also for the numerical methods, because if there exist "strong efficient" points, then they are the only "vectorial efficient" points. Using this conclusion and the abstract construction given by us in (Postolică, V. 1991, 1988) for the splines in the H - locally convex spaces introduced in (Precupanu, $T ., 1969)$ as separated locally convex spaces with any semi - norm satisfying the parallelogram law, linear topological spaces also studied with strictness in (Kramar, E.,1981), it follows that the only best simultaneous and vectorial approximation for each element in the direct sum of any (closed) linear subspace and its orthogonal, with respect to a linear (continuous) operator between two arbitrary $\mathrm{H}$ locally convex spaces, is its spline function. We also note that it is possible to have $S\left(A, K, K_{1}\right)=\varnothing$ and $\operatorname{eff}\left(A, K, K_{1}\right)=A$. Thus, for example, if one considers $X=R^{n}(n \in N, n \geq 2), K=R_{+}^{n}$, $K_{1}=\{(0, \ldots, 0)\}$ and for each real number $c$ one defines $A_{0 c}=\left\{\left(x_{i}\right) \in X: \sum_{i=1}^{n} x_{i}=c\right\}$, $A_{1 c}=\left\{\left(x_{i}\right) \in X: \sum_{i=1}^{n} x_{i} \geq c\right\}, A_{2 c}=\left\{\left(x_{i}\right) \in X: \sum_{i=1}^{n} x_{i} \leq c\right\}$ then it is clear that

$S\left(A_{0 c}, K, K_{1}\right)=S\left(A_{1 c}, K, K_{1}\right)=S\left(A_{2 c}, K, K_{1}\right)=\varnothing$, eff $\left(A_{0 c}, K, K_{1}\right)=\operatorname{MIN}\left(A_{0 c}, K, K_{1}\right)=\operatorname{MAX}\left(A_{0 c}, K, K_{1}\right)=A_{0 c}$, $\operatorname{MIN}\left(A_{1 c}, K, K_{1}\right)=\operatorname{MAX}\left(A_{2 c}, K, K_{1}\right)=A_{0 c}$ so, by extrapolation,

eff $\left(A_{1 c}, K, K_{1}\right)=A_{0 c} \cup\{(+\infty,+\infty, \ldots,+\infty)\}$ and eff $\left(A_{2 c}, K, K_{1}\right)=A_{0 c} \cup\{(-\infty,-\infty, \ldots,-\infty)\}$. At the same time, in the usual real linear space of all sequences composed by real numbers, ordered by the convex cone $K=\left\{\left(x_{n}\right): n \in N^{*}, n \geq 2, x_{n} \geq 0, \forall n \geq 2\right\}$, for $A=\left\{\left(x_{n \alpha}\right): n \in N^{*}, n \geq 2, \alpha>0\right\}$ with

$x_{n \alpha}=(n-1)^{-\alpha}-n^{-\alpha}, n \in N^{*}, n \geq 2, \alpha>0$ and $K_{1}=\{(0,0, \ldots)$.$\} , we have$

eff $\left(A, K, K_{1}\right)=S\left(A, K, K_{1}\right)=\varnothing$. If one considers $E=R^{2}, K=R_{+}^{2}=\left\{(x, y) \in R^{2}: x, y \geq 0\right\}$, $K_{1}=\{(0,0)\}$ and $A=\left\{(x, y) \in R^{2}: x^{2}+y^{2}=1\right\}$ then, $S(A, K)=\varnothing$,

$\operatorname{MIN}(A, K)=\operatorname{MAX}(A, K)=\overparen{A_{1} B_{1}} \cup \overparen{C_{1} D_{1}}$, so eff $(A, K)=\overparen{A_{1} B_{1}} \cup \overparen{C_{1} D_{1}}$, where $A_{1}(1,0), B_{1}(0,1)$ , $C_{1}(-1,0), D_{1}(0,-1)$, with the corresponding extension in any Euclidean space $R^{n}, n \in N^{*}$. Some of the above examples show that, in general, the implication $S\left(A, K, K_{1}\right)=\varnothing \Rightarrow \operatorname{eff}\left(A, K, K_{1}\right)=A$ is not true. However, at least from the numerical methods point of view, we are interested for

Open Problem 3. Characterizations of the equivalence

$$
S\left(A, K, K_{1}\right)=\varnothing \Leftrightarrow \operatorname{eff}\left(A, K, K_{1}\right)=A .
$$

\section{ISAC'S CONES AND THE EFFICIENCY}

Initially, the next concept of convex cone named by its author nuclear, supernormal (Isac, G., 1980, 1981, 1983, 1985) and, by us, Isac's cone (Postolică, V., 2009) was introduced in the following context, being the most appropriate for the general efficiency by optimization and conversely (see, 
for instance, Isac, G., 1980,1981, 1983, 1985, 1994, 1998, 2003; Isac, G., Bahya, A.O., 2002; Isac, G., Postolică, V., 1993, 2005; Isac, G., Tammer, Chr., 2003). As we see, to ensure the existence of the efficient points for a

non - empty set in Hausdorff separated locally convex space, the main reason will be to replace the compactness with the completeness hypothesis imposed on the set and the ordering cone to be an Isac's cone.

Definition 2. (Treves, F., 1967). A locally convex space is any couple (X,Spec $(X))$ which is composed of a real or complex linear space $X$ and a family Spec $(X)$ of seminorms on $X$ such that:

$$
\chi p \in \operatorname{Spec}(X), \forall \chi \in R_{+}, p \in \operatorname{Spec}(X) ;
$$

(ii) if $p \in \operatorname{Spec}(X)$ and $q$ is an arbitrary seminorm on $X$ such that $q \leq p$, then $q \in \operatorname{Spec}(X)$;

(iii) $\quad \sup \left(p_{1}, p_{2}\right) \in \operatorname{Spec}(X), \forall p_{1}, p_{2} \in \operatorname{Spec}(X)$ where

$$
\sup \left(p_{1}, p_{2}\right)(x)=\sup \left(p_{1}(x), p_{2}(x)\right), \forall x \in X \text {. }
$$

It is well known that whenever such a family as this $\operatorname{Spec}(X)$ is given on a real vector space $X$, there exists a locally convex topology $\tau$ on $X$ such that $(X, \tau)$ is a topological linear space and a seminorm $p$ on $X$ is

$\tau$ - continous iff $p \in \operatorname{Spec}(X)$. A non-empty subset $\mathrm{B}$ of $\operatorname{Spec}(X)$ is a base for it if for every $p \in \operatorname{Spec}(X)$ there exist $\chi>0$ and $q \in \mathrm{B}$ such that $p \leq \chi q$ and $(X, \tau)$ is a Hausdorff locally convex space iff $\operatorname{Spec}(X)$ has a base B, named a Hausdorff base, with the property that

$\{x \in X: p(x)=0, \forall p \in \mathrm{B}\}=\{\theta\}$ where $\theta$ is the null vector in $X$. In this paper we will suppose that the space $(X, \tau)$, sometimes denoted by $X$, is a Hausdorff locally convex space. We recall that every non - empty subset $K$ of $X$ satisfying the following properties: $K+K \subseteq K$ and $\chi K \subseteq K, \forall \chi \in R_{+}$is named convex cone. If, in addition, $K \cap K=\{\theta\}$, then $K$ is called pointed. Clearly, any pointed convex cone $K$ in $X$ generates an ordering on $X$ defined by $x \leq y(x, y \in X)$ iff $y-x \in K$. If $X^{*}$ is the dual of $X$, then the dual cone of $K$ is defined by $K^{*}=\left\{x^{*} \in X^{*}: x^{*}(x) \geq 0, \forall x \in K\right\}$ and its corresponding polar is $K^{0}=-K^{*}$. It is well known that a pointed convex cone $K \subset(X, \operatorname{Spec}(X))$ is normal with respect to the topology defined by $\operatorname{Spec}(X)$ if it fulfils one of the next equivalent assertions:

(i) there exists a base $\Omega$ of neighborhoods for the origin $\theta$ in $X$ such that

$$
V=(V+K) \cap(V-K), \forall V \in \Omega ;
$$

(ii) there exists a base $\mathrm{B}$ of $\operatorname{Spec}(X)$ with $p(x) \leq p(y), \forall x, y \in K, x \leq y, \forall p \in B$;

(iii) for any two nets $\left\{x_{i}\right\}_{i \in I},\left\{y_{i}\right\}_{i \in I} \subset K$ with $\theta \leq x_{i} \leq y_{i}, \forall i \in I$ and $\lim y_{i}=\theta$

it follows that $\lim x_{i}=\theta$. In particular, a convex cone $K$ is normal in a normed linear space $(E,\|\|$.$) iff there exists t \in(0, \infty)$ such that $x, y \in E$ and $y-x \in K$ implies that $\|x\| \leq t\|y\|$. It is well known (Treves, F., 1967) that, the concept of normal cone together with its natural generalizations, represents the most important notion in the Theory and the Applications of the Convex Cones in the general Topological Ordered Vector Spaces. Thus, for example, for every separated locally convex space $(X, \operatorname{Spec}(X))$ and any closed normal cone $K \subset(X, \operatorname{Spec}(X))$ we have $X^{*}=K^{*}-K^{*}$ (for more details see Hyers, D., H., Isac, G., Rassias,T.M., 1997; Isac, G., 1980). Each pointed convex cone $K \subset(X, \operatorname{Spec}(X))$ for which there exists a non - empty, convex 
bounded set $B \subset X$ such that $\theta \notin \bar{B}$ and $K=\bigcup_{\chi \geq 0} \chi B$ is called well-based. A cone $K \subset(X, \operatorname{Spec}(X))$ is well - based iff there exists a base $B=\left\{p_{i}\right\}_{i \in I}$ of $\operatorname{Spec}(X)$ and a linear continuous functional $f \in K^{*}$ such that for every $p_{i} \in B$ there exists $c_{i}>0$ with $c_{i} p_{i}(x) \leq f(x), \forall x \in K$ (Hyers, D., H., Isac, G., Rassias,T.M., 1997; Isac, G., 1980). Evidently, every well - based cone is a normal cone, but, in general, the converse is not true, as we can see in the examples below, starting from the next basic notion.

Definition 3. (Isac, G., 1981,1983). In a Hausdorff locally convex space (X, Spec(X)), a pointed convex cone $K \subset X$ is nuclear (supernormal) with respect to the topolgy induced by $\operatorname{Spec}(X)$ if there exists a base $B=\left\{p_{i}\right\}_{i \in I}$ of $\operatorname{Spec}(X)$ such that for every $p_{i} \in B$ there exists $f_{i} \in X^{*}$ with

$$
p_{i}(x) \leq f_{i}(x), \forall x \in K .
$$

Remark 5. Initially, the regretted Professor George Isac considered that Treves'definition was more scientifically productive for its cones. Afterwards, he accepted our proposal. Thus, for the first time, we called any such as this cone "Isac's cone" published and officially recognized in (Postolică, V., 2009). For this reason, we will call these cones"supernormal" or "Isac's cones" as folows, taking into account that the above definition of any locally convex space is equivalent with the following: let $X$ be a real or complex linear space and let $P=\left\{p_{\alpha}: \alpha \in A\right\}$ be a family of seminorms defined on $X$. For every $x \in X, \varepsilon>0$ and $n \in N^{*}$ let

$V\left(x ; p_{1}, p_{2}, \ldots, p_{n} ; \varepsilon\right)=\left\{y \in X: p_{\alpha}(y-x)<\varepsilon, \forall \alpha=\overline{1, n}\right\}$ be the family

$\left.\triangle_{0}(x)=\left\{V\left(x ; p_{1}, p_{2}, \ldots, p_{n} ; \varepsilon\right): n \in N^{*}, p_{\alpha} \in P, \alpha=\overline{1, n}, \varepsilon>0\right\}\right\}$, with the following

properties :

$\left(\mathrm{V}_{1}\right) x \in V, \forall V \in \Delta_{0}(x)$;

$\left(\mathrm{V}_{2}\right) \forall V_{1}, V_{2} \in \Delta_{0}(x), \exists V_{3} \in \Delta_{0}(x): V_{3} \subseteq V_{1} \cap V_{2}$;

$\left(\mathrm{V}_{3}\right) \forall V \in \Delta_{0}(x), \exists U \in \Delta_{0}(x), U \subseteq V$ such that $\forall y \in U, \exists W \in \Delta_{0}(y)$ with $W \subseteq V$.

Therefore, $\triangle_{0}(\mathrm{x})$ is a base of neighborhoods for $x$ and taking $\triangle(x)=\left\{V \subseteq X: \exists U \in \triangle_{0}(\mathrm{x})\right.$ with $U \subseteq V\}$, the set

$\tau=\{D \subseteq X: D \in \Delta(x), \forall x \in D\} \cup\{\varnothing\}$ is the locally convex topology generated by the family $P$. Obviously, the usual operations which induce the structure of linear space on $X$ are continuous with respect to this topology. The corresponding topological space $(X, \tau)$ is a Hausdorff locally convex space iff the family $P$ is sufficient, that is, $\forall x_{0} \in X \backslash\{\theta\}, \exists p_{\alpha} \in P$ with $p_{\alpha}\left(x_{0}\right) \neq 0$. In this context, a convex cone $K \subset X$ is an Isac's cone iff

$$
\forall p_{\alpha} \in P, \exists f_{\alpha} \in X^{*}: p_{\alpha}(x) \leq f_{\alpha}(x), \forall x \in K
$$

This is an equivalent definition of Isac's cones in separated locally convex spaces. The best special, refined and non - trivial Isac's cones class associated to normal cones in Hausdorff locally convex spaces was introduced and studied in (Isac, G., Bahya,A., O., 2002) as the full nuclear cones family defined as follows: if $(X, \operatorname{Spec}(X))$ is an arbitrary locally convex space, $B \subset \operatorname{Spec}(X)$ is a Hausdorff base of $\operatorname{Spec}(X)$ and $K \subset X$ is a normal cone, then for any mapping $\varphi: B \rightarrow K^{*} \backslash\left\{\theta^{*}\right\}$ one says that the set 
$K_{\varphi}=\{x \in X: p(x) \leq \varphi(p)(x), \forall p \in B\}$ is the full nuclear cone associated to $K$ whenever $K_{\varphi} \neq\{\theta\}$. In fact, $K$ is supernormal iff there exists

$\varphi: P \rightarrow \mathrm{K}^{*} \backslash\{0\}$ such that $\mathrm{K} \subseteq \mathrm{K}_{\varphi}$ (Poposition 3.1 given by (Isac, G. and Postolică, V., 2005). In the above equivalent context, if $\varphi: P \rightarrow K^{*}$ is a function, then the convex cone $K_{\varphi}=\left\{x \in X: p_{\alpha}(x) \leq \varphi\left(p_{\alpha}\right)(x), \forall p_{\alpha} \in P\right\}$ is the full nuclear cone associated to $\mathrm{K}, P$ and $\varphi$. In a real normed linear space $(E,\|\|$.$) , a non - empty set T \subset E$ is called a Bishop - Phelps cone if there exists $y^{*}$ in the usual dual space $E^{*}$ of $E$ and $\alpha \in(0,1]$ such that

$T=\left\{y \in E: \alpha\|y\| \leq y^{*}(y)\right\}$. Following the applications of such as these cones in Nonlinear Analysis, including the Optimization Programs with

Vector - Valued Objective Mappings, one concludes that, for Haudorff locally convex spaces, the full nuclear cones are natural generalizations of Bishop - Phelps Cones in Normed Vector Spaces.

The beginning and the considerations in Section 4 of (Isac, G., Bahya, A. O., 2002) suggested us to consider for each function $\varphi: P \rightarrow K^{*} \backslash\{0\}$ the full nuclear cone

$K_{\varphi}=\left\{x \in X: p_{\alpha}(x) \leq \varphi\left(p_{\alpha}\right)(x), \forall p_{\alpha} \in P\right\}$ in order to give the next generalization of Theorem 7 in (Isac,G., Bahya, A.O., 2002). It represents an extension of the main result indicated by (Isac, G., Postolică, V., 2005), an important link between the efficiency, the full nuclear cones, strong optimization and a significant result concerning the multiple scalarization for this general concept of the efficiency.

Theorem 2. If $K$ is any Isac's cone in every Hausdorff locally convex space $\left(X, P=\left\{p_{\alpha}: \alpha \in A\right\}\right)$, then

(i)

$$
\begin{aligned}
& \text { (i) } \operatorname{MIN}\left(A, K, K_{1}\right)=\bigcup_{\substack{a \in A \\
\varphi \in P \rightarrow K^{*} \backslash\{0\}}} \operatorname{SMIN}\left(A \cap\left(a-K-K_{1}\right), K_{\varphi}\right) \text {; } \\
& \text { (ii) } \operatorname{MAX}\left(A, K, K_{1}\right)=\bigcup_{\substack{a \in A \\
\varphi \in P \rightarrow K^{*} \backslash\{0\}}} \operatorname{SMAX}\left(A \cap\left(a+K+K_{1}\right), K_{-\varphi}\right) \text {; }
\end{aligned}
$$

$$
\operatorname{eff}\left(A, K, K_{1}\right)=\bigcup_{\substack{a \in A \\ \varphi \in P \rightarrow K^{*} \backslash\{0\}}}\left[\operatorname{SMIN}\left(A \cap\left(a-K-K_{1}\right), K_{\varphi}\right) \cup \operatorname{SMAX}\left(A \cap\left(a+K+K_{1}\right), K_{-\varphi}\right)\right] .
$$

for any non - empty subset $K_{1}$ of $K$.eff $\left(A, K, K_{1}\right)=\operatorname{eff}(A, K)$

Proof. It follows, mutatis mutandis, the same line as for Theorem 4.3 given by Isac, G.and Postolică, $V$. in 2005.

Remark 6. To obtain (ii) from (i) it is not about of a simple formality by replacing $K$ with $(-K)$, since $(-K)_{\varphi}=-K_{-\varphi}$. If $\theta \notin K_{1}$, then $a_{0} \in e f f\left(A, K, K_{1}\right)$ implies that $A \cap\left(a_{0}-K-K_{1}\right)=\varnothing$. Therefore, it is not possible to have $a_{0} \in S\left(\varnothing, K_{\varphi}\right)$. In the case of $\theta \in K_{1}$, then eff $\left(A, K, K_{1}\right)=e f f(A, K)$ and

$a_{0} \in e f f(A, K)$ iff $A \cap\left(a_{0}-K\right)=\left\{a_{0}\right\}$, so in the right member of the first proved inclusion it can be selected any convex cone, not necessary $K_{\varphi}$. The hypothesis $K \subseteq K_{\varphi}$ imposed upon the convex cone $K$ and automatically satisfied since $K$ is an Isac's cone was used only to prove the next inclusion, with its natural projection for $\operatorname{MAX}\left(A, K, K_{1}\right)$ : 


$$
\operatorname{MIN}\left(A, K, K_{1}\right) \subseteq \bigcup_{\substack{a \in A \\ \varphi: P \rightarrow K^{*} \backslash\{0\}}} \operatorname{SMIN}\left(A \cap\left(a-K-K_{1}\right), K_{\varphi}\right)
$$

Corollary 2.1. For every non-empty subset A of any Hausdorff locally convex space ordered by an arbitrary Isac's cone $K$ with its dual cone $K^{*}$ we have

(i) $\operatorname{MIN}(A, K)=\bigcup_{\substack{a \in A \\ \varphi: P \rightarrow K^{*} \backslash\{0\}}} \operatorname{SMIN}\left(A \cap(a-K), K_{\varphi}\right)$;

(ii) $\operatorname{MAX}(A, K)=\bigcup_{\substack{a \in A \\ \varphi: P \rightarrow K^{*} \backslash\{0\}}} \operatorname{SMAX}\left(A \cap(a+K), K_{-\varphi}\right)$;

(iii)

$$
\text { eff }(A, K)=\bigcup_{\substack{a \in A \\ \varphi \in P \rightarrow K^{*} \backslash\{0\}}}\left[\operatorname{SMIN}\left(A \cap(a-K), K_{\varphi}\right) \cup \operatorname{SMAX}\left(A \cap(a+K), K_{-\varphi}\right)\right] .
$$

Remark 7.The hypothesis of the above theorem involves $K$ to be pointed. Consequently, $\theta \in K_{1}$ iff $\theta \in K+K_{1}$. If $a_{0} \in S\left(A \cap\left(a-K-K_{1}\right), K_{\varphi}\right)$ for some $\varphi: P \rightarrow K^{*}$ and $a \in A$ with $a_{0}=a-k-k_{1}, k \in K, k_{1} \in K_{1}$, then $K \cap\left(a_{0}-A\right)=\{\theta\}$ because $A \cap\left(a-K-K_{1}\right) \subseteq a_{0}+K_{\varphi}$ in any such a case as this. Indeed, let $x \in K \cap\left(a_{0}-A\right)$ be an arbitrary element. Then, $a_{0}-x \in A$ and $a_{0}-x=a-k-k_{1}-x \in a-K-K_{1}$.Therefore, $a_{0}-x \in a_{0}+K_{\varphi}$, that is, $x \in K_{\varphi}$. For every $p_{\alpha} \in P$ we have $p_{\alpha}(-x) \leq \varphi\left(p_{\alpha}\right)(-x)=-\varphi\left(p_{\alpha}\right)(x) \leq 0$. Since $\mathrm{p}_{\alpha}$ was arbitrary chosen in $P$ and $X$ is a Hausdorff locally convex space, it follows that $\mathrm{x}=\theta$.

Remark 8. Clearly, the announced theorem represents a significant result concerning the possibilities of multiple scalarization for the study of the efficiency programs in Hausdorff separated locally convex spaces, as we can see also in the final comments of (Isac,G., Bahya, A.O., 2002) for the particular cases of Hausdorff locally convex spaces ordered by closed, pointed and normal cones.

Open Problem 4. Apply the above research taking in place of $K_{1}$ any non-empty subset of an arbitrary ordered linear space, under proper hypotheses.

Remark 9. In the next considerations, we offer significant examples and adequate remarks on Isac's cones with the mention that for the existence of the efficient points and important properties of the efficient points sets in separated locally convex spaces ordered by (weak) such as these convex cones, through the agency of the (weak) completeness instead of compactness, the reader is referred to (Isac, G., 1981, 1983, 1985, 1994, 1998; Isac, G., Postolică, V., 1993; Postolică, V., 1993,1994,1995, 1997, 1999, 2001, 2002, 2009; Truong, X. D. H., 1994 and the references therein).

Theorem 3. (Bahya, A. O., 1989). A convex and normal cone $\mathrm{K}$ in a Hausdorff locally convex space is supernormal if and only if every net of $\mathrm{K}$ weakly convergent to zero converges to zero in the locally convex topology.

Let us consider some pertinent examples which can be also found in (Postolică, V., 2014, 2015, 2016).

1. Any pointed, convex cone, in every Euclidean space $R^{k}\left(\mathrm{k} \in \mathrm{N}^{*}\right)$ is an Isac's cone.

2. In every locally convex space, any well-based convex cone is an Isac's cone.

3. A convex cone is an Isac's cone in a normed linear space if and only if it is well-based.

4. Let $n \in N^{*}$ be arbitrary fixed and let $Y$ be the space of all real symmetric $(n, n)$ matrices ordered by the pointed, convex cone

$\mathrm{C}=\left\{\mathrm{A} \in \mathrm{Y}: \mathrm{x}^{\mathrm{T}} \mathrm{Ax} \geq 0, \forall x \in R^{n}\right\}$. Then, $Y$ is a real Hilbert space with respect to the scalar product defined by $\langle A, B\rangle=\operatorname{trace}(A$. $B$ ) for all $A, B \in Y$ and $C$ is well-based by $B=\{A \in C:\langle A, I\rangle=1\}$, where I denotes the identity matrix. 
5. Every pointed, locally or weakly locally compact convex cone in any Hausdorff locally convex space is an Isac's cone.

6. A convex cone is an Isac's cone in a nuclear space (Pietch, A., 1972) if and only if it is a normal cone.

7. In any Hausdorff locally convex space, a convex cone is an weakly Isac's cone if and only if it is weakly normal.

8. In $L^{p}([a, b]),(p \geq)$, the convex cone $K_{p}=\left\{x \in L^{p}([a, b]): x(t) \geq 0\right.$ almost everywhere $\}$ is an Isac's cone if and only if $p=1$, being well-based in this case by the set $B=\left\{x \in K_{1}: \int_{\mathrm{a}}^{\mathrm{b}} \mathrm{x}(\mathrm{t}) \mathrm{dt}=1\right\}$. Indeed, if $p>1$, then the sequence $\left(x_{n}\right)$ defined by

$$
x_{n}(t)=\left\{\begin{array}{l}
n^{1 / p}, a \leq t \leq a+(b-a) / 2 n \\
0, a+(b-a) / 2 n<t \leq b
\end{array} \quad n \in \mathbf{N}\right.
$$

converges to $O$ in the weak topology but not in the usual norm topology. Therefore, by virtue of Theorem $1, K_{p}$ is not an Isac's cone. Generally, for every $p>1, K_{p}$ has a base $B=\left\{x \in K_{p}: \int_{\mathrm{a}}^{\mathrm{b}} \mathrm{x}(\mathrm{t}) \mathrm{dt}=1\right\}$ which is unbounded and any cone generated by a closed and bounded set $B_{t}=\left\{x \in B: \int_{\mathrm{a}}^{\mathrm{b}}|\mathrm{x}(\mathrm{t})|^{\mathrm{p}} \mathrm{dt} \leq \mathrm{t}\right\}$ with $t \geq 0$ is certainly an Isac's cone. A similar result holds for $L^{p}(R)$. Thus, if we consider a countable family $\left(A_{n}\right)$ of disjoint sets which covers $R$ such that $\mu\left(A_{n}\right)=1$ for all $n$ in $N$, where $\mu$ is the Lebesgue measure, then the sequence $\left(y_{n}\right)$ given by $y_{n}(t)=1$ if $t \in A_{n}$ and $y_{n}(t)=0$ for $t \in R \backslash A_{n}$ converges weakly to zero while it is not convergent to zero in the norm topology. Taking into account the above theorem, it follows that the usual positive cone in $L^{p}(R)$ is not an Isac's cone if $p>1$, that is, it is not well - based in all these cases. However, these cones are normal for every $p \geq 1$. The same conclusion concerning the non - supernormality is valid for the positive orthant convex cones in the usual Orlicz spaces.

9. In $l^{p}(p \geq 1)$ equipped with the usual norm $\|\cdot\|_{\mathrm{p}}$ the positive cone

$C_{p}=\left\{\left(x_{n}\right) \in l^{p}: x_{n} \geq 0\right.$ for all $\left.n \in N\right\}$ is also normal with respect to the norm topology, but it is not an Isac's cone excepting the case $p=1$. Indeed, for every $p>1$, the sequence $\left(e_{n,}\right)$ having 1 at the $n-t h$ coordinate and zeros elsewhere converges to zero in the weak topology, but not in the norm topology and, by virtue of Theorem 3, it follows that $C_{p}$ is not an Isac's cone. For $p=1, \mathrm{C}_{\mathrm{p}}$ is well - based by the set $\mathrm{B}=\left\{\mathrm{x} \in \mathrm{C}_{1}:\|\mathrm{x}\|_{1}=1\right\}$ and Proposition 5 (Isac, G., 1983) ensures that it is an Isac's cone. In this case, the locally convex topology defined by the seminorms $p_{n}\left(\left(x_{k}\right)\right)=\sum_{\mathrm{k}=0}^{\mathrm{n}}\left|\mathrm{x}_{\mathrm{k}}\right|$ for every $\left(x_{k}\right)$ in $l^{l}$ and $n$ $\in N$ is weaker than its usual weak topology. Consequently, the usual positive cone remains an Isac's cone with respect to this topology (now it is normal in a nuclear space and one applies Proposition 6 of (Isac, G., 1983) but it is not well-based. We now recall the concept of $H$ - locally convex space introduced by Precupanu, T. in 1969: any Hausdorff locally convex space with the seminorms satisfying the parallelogram law. Taking into account it and the property that every nuclear space is also a H-locally convex space with respect to an equivalent system of seminorms (Pietch, A., 1972), the above example shows that in a H-locally convex space a proper convex cone may be an Isac's cone without to be well - based. Moreover, if we consider in $l^{2}$ the $H$ - locally convex topology induced by the seminorms

$$
\tilde{\mathrm{p}}_{\mathrm{n}}\left(\left(\mathrm{x}_{\mathrm{k}}\right)\right)=\left(\sum_{\mathrm{i} \geq \mathrm{n}}\left|\mathrm{x}_{\mathrm{i}}\right|^{2}\right)^{1 / 2}, \mathrm{n} \in \mathrm{N},\left(\mathrm{x}_{\mathrm{k}}\right) \in \ell^{2}
$$

then the convex cone $C_{2}=\left\{\left(x_{k}\right) \in l^{2}: x_{k} \geq 0\right.$ for all $\left.k \in N\right\}$ is normal in the 
$H$ - locally convex space $\left(l^{2},\left\{\tilde{\mathrm{p}}_{\mathrm{n}}\right\}_{\mathrm{n} \in \mathrm{N}}\right)$, but it is not a supernormal cone because the same sequence $\left(e_{k}\right)$ is weakly convergent to zero while $\left(\overline{\mathrm{p}}_{\mathrm{n}}\left(\mathrm{e}_{\mathrm{k}}\right)\right)$ is convergent to 1 for each $n \in N$ and one applies again Theorem 3. Another interesting example of normal cone in a $H$-locally convex space which is not Isac's cone is the usual positive cone in the space $L^{2}$ loc $(R)$ of all functions from $R$ to $C$ which are square integrable over any finite interval of $R$, endowed with the system of seminorms $\left\{\overline{\mathrm{p}}_{\mathrm{n}}: \mathrm{n} \in \mathrm{N}\right\}$ defined by $\overline{\mathrm{p}}_{\mathrm{n}}(\mathrm{x})=\left(\int_{-\mathrm{n}}^{\mathrm{n}}|\mathrm{x}(\mathrm{t})|^{2} \mathrm{dt}^{1 / 2}\right)$ for every $x$ in $L_{\text {loc }}^{2}(R)$. In this case, the sequence $\left(x_{k}\right)$ given by:

$$
\mathrm{x}_{\mathrm{k}}(\mathrm{t})=\left\{\begin{array}{l}
0, \mathrm{t} \in(-\infty, 0) \cup(1 / \mathrm{k},+\infty) \\
\sqrt{\mathrm{k}}, \mathrm{t} \in[0,1 / \mathrm{k}]
\end{array}\right.
$$

converges weakly to zero, but it is not convergent in the $H$ - locally convex topology. The results follows by Theorem 3. It is clear that every weak topology is a H-locally convextopology and, in these cases, the supernormality of convex cones coincides with the normality thanks to the Corollary of Proposition 2 in (Isac, G., 1983).

10. In the space $C([a, b])$ of all continuous, real valued functions defined on every non - trivial, compact interval $[a, b]$ equipped with the usual supremum norm the convex cone

$K=\{x \in C([a, b]): x$ is concave, $x(a)=x(b)=0$ and $x(t) \geq 0$ for all $t \in[a, b]\}$ is an Isac's cone, being well-based by the set $\left\{x \in K: x\left(t_{0}\right)=1\right\}$ for some arbitrary $t_{0} \in[a, b]$. The hypothesis that all $x \in K$ are concave is essential for the supernormality.

11. The convex cone of all non-negative sequences in the space of all absolutely convergent sequences is the dual of the usual positive cone in the space of all convergent sequences. Consequently, it has a weak star compact base and hence it is a weak star Isac's cone.

12. In $l^{p}$ or in $c_{0}$ equipped with the supremum norm, the convex cone consisting of all sequences having all partial sums non - negative is not normal, hence it is not supernormal.

13. In every Hausdorff locally convex space any normal cone is an Isac's cone with respect to the weak topology.

14. In every locally convex lattice which is a $(L)$ - space the ordering cone is supernormal (see also Example 7 given by Isac, G. in 1994).

15. If we consider the space of all locally integrable functions on a locally compact space $Y$ with respect to a Radon measure $\mu$ endowed with the topology induced by the family of seminorms $\left\{p_{A}\right\}$ where $p_{A}(f)=\int_{A}|\mathrm{f}(\mathrm{x})| \mathrm{d} \mu$ for every non-empty and compact subset $A$ of $Y$ and every locally integrable function $f$, then the convex cone $K=\{f: f(x) \geq 0, x \in Y\}$ is supernormal.

16. If $Z$ is any locally convex lattice ordered by an arbitrary convex cone $K$ and $Z^{*}$ is its topological dual ordered by the corresponding dual cone $K^{*}$, then the cone $K$ is an Isac's cone with respect to the locally convex topology defined on $Z$ by the neighbourhood base at the origin $\left\{[-f, f]^{\circ}\right\}_{f}$.

17 In every regular vector space $(E, K)$ (that is, the order dual $E^{*}$ separates the points of $E$ ) with the property that $E=K-K$, the convex cone $K$ is supernormal with respect to the topology defined in the preceding example.

18. Any semicomplete cone in a Hausdorff locally convex space is an Isac's cone (see Example 11 of Isac, G., 1994).

Remark 10. Clearly, if a convex cone $K$ is supernormal in a normed space, then $K$ admits a strictly positive, linear and continuous functional, that is, there exists a linear, continuous functional $f$ such that $f(k) \geq 0$ for all $\mathrm{k} \in \mathrm{K} \backslash\{\theta\} . k \in K$. Generally, the converse is not true even in a Banach space as we can see in the following examples. 
19. If one considers in the usual space $l^{p} \quad(1 \leq p \leq \infty)$ the convex cone $K$ $=\ell_{+}^{\mathrm{p}}=\left\{\mathrm{x}=\left(\mathrm{x}_{\mathrm{i}}\right) \in \ell^{\mathrm{p}}: \mathrm{x}_{\mathrm{i}} \geq 0\right.$ for every $\left.\mathrm{i} \in \mathrm{N}\right\}$ of infinite vectors with non-negative components, then the functional $\varphi$ defined by $\varphi(\mathrm{k})=\sum_{\mathrm{i}=1}^{\infty} \mathrm{k}_{\mathrm{i}}$ for any $k=\left(k_{i}\right) \in l^{p}$ is linear, continuous and strictly positive. But, as we have seen in the above considerations (Example 9), this cone is supernormal if and only if $p=1$.

20. Let $K$ be the usual positive cone

$L^{p}{ }_{+}=\left\{x \in L^{p}([a, b]): x(t) \geq 0\right.$ almost everywhere $\}$ in $L^{p}([a, b], \quad(1 \leq p \leq \infty)$. Then, the linear and continuous functional $\psi$ on $L^{p}([a, b])$ given by $\psi(\mathrm{x})=\int_{\mathrm{a}}^{\mathrm{b}} \mathrm{x}(\mathrm{t}) \mathrm{dt}$ for every $x \in L^{p}([a, b])$ is strictly positive on $K$ while $K$ is supernormal (see the above Example 8) if and only if $p=1$. The same conclusion is valid for the space $L^{p}(R)((1 \leq p \leq \infty))$. Therefore, $l^{l}{ }_{+}$and $L^{l}{ }_{+}$are Isac's cones, with empty topological interiors, and for every $p \in(1,+\infty)$ it follows that $l^{p}{ }_{+}$and

$L^{p}{ }_{+}$are normal cones, with empty interiors, which are not supernormal. Hence, these convex cones are not well based. Very simple examples of Isac's cones having non-empty topological interiors are $R_{+}{ }^{n}\left(n \in N^{*}\right)$.

Remark 11. In the order complete vector lattice $B([a, b])$ of all bounded, real valued functions on $a$ compact non - singleton interval $[a, b]$ endowed with its usual norm the standard positive cone

$K=\{u \in B([a, b]): u(t) \geq 0$ for all $t \in([a, b])\}$ is normal but it has not a base, that is, it is not an Isac's cone. However, this cone has non-empty interior. If we consider the linear space $l^{l}$ endowed with the separated locally convex topology generated by the family $\left\{p_{n}: n \in N\right\}$ of seminorms defined by $\mathrm{p}_{\mathrm{n}}(\mathrm{x})=\sum_{\mathrm{k}=0}^{\mathrm{n}}\left|\mathrm{x}_{\mathrm{k}}\right|$ for every $\mathrm{x}=\left(x_{k}\right) \in l^{l}$, then the convex cone

$K=\left\{x=\left(x_{k}\right) \in l^{l}: x_{k} \geq 0\right.$ whenever $\left.k \in N\right\}$ is an Isac's cone but it is not

well-based.

Open Problem 5. We introduce Isac's sets in the following area:let $M$ be a non-empty subset of $X$ and let cone $(M)$ be the convex cone generated by $M$. Obviously, the quality of Isac's cone for cone $(M)$ is equivalent with the fact that each seminorm is majorized by at least a linear and continuous functional on M. Therefore, it is natural to call it "Isac set". So, another open problem is to discover the properties of these sets.

In synthesis, the position of Isac's Cones in the world of the Ordered Vector Spaces is the following:

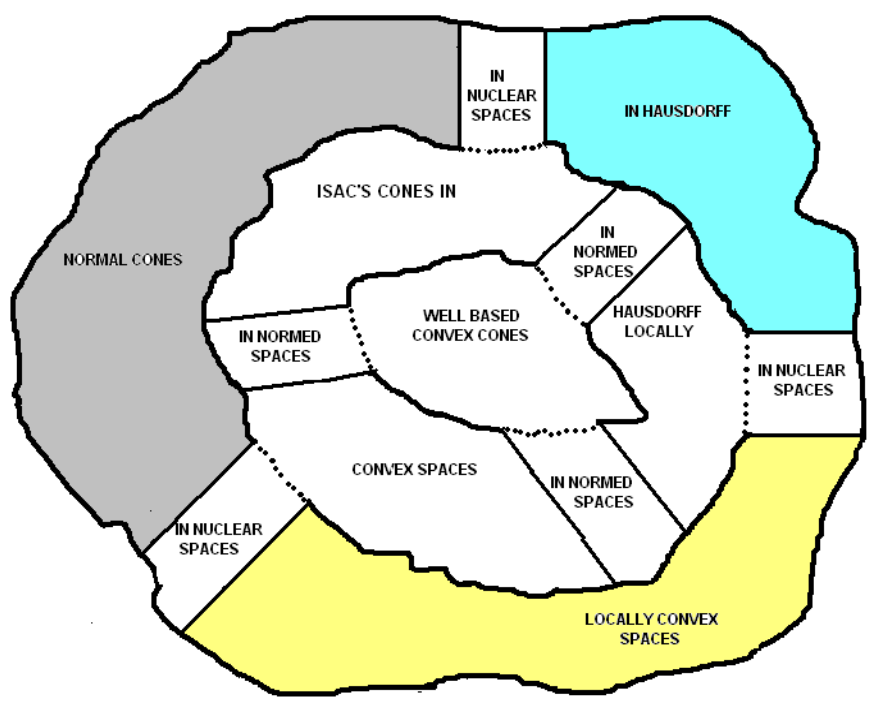




\section{SOME CONCLUSIONS}

The natural context of supernormality (nuclearity) for convex cones is any separated locally convex space. Isac, G. introduced the concept of "nuclear cone" in 1981, published it in 1983 and he showed that in a normed space, a convex cone is nuclear if and only if it is well-based or equivalently iff it is "with plastering", the last concept being defined by Krasnoselski, M. A. in fifties (see ,for example, Krasnoselski, M. A., 1964 and so on). Such a convex cone was initially called "nuclear cone" by Isac, G. (1981) because in every nuclear space (Pietch, A., 1972), any normal cone is a nuclear cone in Isac's sense (Proposition 6 of Isac, G., 1983). Later on, since the nuclear cone introduced by Isac appears as a reinforcement of the normal cone, it was called supernormal. Following the friendly approval of Professor Isac, we called and published it as "Isac's Cone" in 2009. The class of supernormal cones in Hausdorff locally convex spaces was initially imposed by the theory and the applications of the efficient (Pareto minimum type) points (especially existence conditions based on completeness instead of compactness were decisive together with the main properties of the efficient points sets). Afterwards, the study of critical points for dynamical systems and conical support points completed its importance, very well illustrated by final results, examples and comments, specified in our references and in all connected papers on this subject. It is also very significant to mention again that the concept of supernormality introduced by Isac, G. (1981) is not a simple generalization of the corresponding notion defined in normed linear spaces by Krasnoselski, M. A. and his colleagues in the fifties. Thus, for example, Isac's supernormality attached to the convex cones has his sense in every Hausdorff locally convex space identically with the well known Grothendieck's nuclearity. By analogy with the fact that a normed space is nuclear in Grothendieck's sense if and only if it is isomorphe with an usual Euclidean space, a convex cone is supernormal in a normed space iff it is well-based, that is, it is generated by a convex bounded set which does not contain the origin in its closure. Beside Pareto type optimization, we also mention Isac's significant contributions, through the agency of supernormal cones, to the convex cones in product linear spaces and Ekeland's variational type principles (Isac.G.,2003; Isac,G.,Tammer,Chr., 2003). Therefore, the more appropriate background for Isac's cones is any separated locally convex space. On the other hand, the extension in real or complex vector spaces of this notion can be done since any real or complex vector space can be considered as a locally convex space with respect to the locally convex topology generated by the family of the seminorms originated in the Minkowski functionals associated to the algebraic and geometric concepts of the convex hulls for the balanced (circled) and absorbing sets containing its origin (Postolică, V., 2014). The behaviour of Isac's cones in some variable locally convex topologies, especially in duality, is also interesting (Postolică,V., 2015). To draw a conclusion, the Best Ordering Mathematical Structure discovered till now for the study of the General Efficiency and its particular case represented by the Vectorial Optimization with the coresponding Applications is represented by Isac's Cones.

Definition 4. (Postolică, $V$., 1995). A nonempty set $B$ of a topological vector space $X$ ordered by a convex cone $K$ is called $K$ - bounded if there exists $B_{0} \subseteq X$, bounded, so that $B \subseteq B_{0} \pm K$ and it is $K$ - closed if its conical extension $B \pm \bar{K}$ is closed, where $\bar{K}$ signifies the topological closure of $K$.

A synthesis concerning the existence and significant properties of the efficient points are given in the next theorem.

Theorem 4. (Postolică, V., 1995, 1997, 2008 ).

(i) if $K$ is an arbitrary Isac's cone in any Hausdorff locally convex space $X, A, B \subseteq X$ are every non-empty subsets positioned by $A \subseteq B \subseteq A+K(A \subseteq B \subseteq A-K)$ and $B \cap\left(A_{0}-K\right)$ ( $\left.B \cap\left(A_{0}+K\right)\right)$ is a bounded and complete set for at least one non-empty set $A_{0} \subseteq A$, then $\operatorname{MIN}(A, K) \neq \varnothing(\operatorname{MAX}(A, K) \neq \varnothing)$;

(ii) if $X$ is a quasi - complete Hausdorff separated locally convex space and $K$ is a closed Isac's cone in $X$, then for any $K$ - bounded and $K$ - closed

non - empty set $A$ of $X$ we have $\operatorname{MIN}(A, K) \neq \varnothing, A \subseteq M I N(A, K)+K$

( $A \subseteq \operatorname{MAX}(A, K)-K)$ (the domination property), $\operatorname{MIN}(A, K)+K=A+K$ 
$(\operatorname{MAX}(A, K)-K=A-K)$ and $\operatorname{MIN}(A, K)(\operatorname{MAX}(A, K))$ is $K$ - bounded and

$K$ - closed;

(iii) according to the same hypotheses from (ii), $\operatorname{MIN}(A, K) \neq \varnothing(\operatorname{MAX}(A, K) \neq \varnothing)$ for any non empty subset $A$ with $B \cap\left(A_{0}-K\right)\left(B \cap\left(A_{0}+K\right)\right) K$ - bounded and $K$ - closed set whenever $A_{o} \subseteq A \subseteq X$ and $A \subseteq B \subseteq A+K(A \subseteq B \subseteq A-K)$, with the corresponding implications for $\operatorname{Eff}(A, K)$.

Open Problem 6. Extend the above theorem for the approximate efficient points given by non-empty subsets $K_{1}$ of $K$. If eff $\left(A, K, K_{1}\right) \neq \varnothing$, then there exist a Hausdorff locally convex space $Y$, an Isac's cone $K_{0}$ in $Y, \varnothing \neq K_{01} \subseteq K_{0}$ and a non - empty set $A_{0} \subset Y$ with eff $\left(A, K, K_{1}\right)=$ eff $\left(A_{0}, K_{0}, K_{01}\right)$ (or, at least, eff $\left(A, K, K_{1}\right)$ to be dense in eff $\left.\left(A_{0}, K_{0}, K_{01}\right)\right)$ ?

\section{EFFICIENCY AND CHOQUET BOUNDARIES}

The main connection between the general efficiency and the Potential Theory is briefly presented through the agency of the Choquet boundaries.

Definition 5.( Postolică, $V ., 2008)$ A real function $f: X \rightarrow R$ is called $\left(K+K_{1}\right)$ - increasing( $K+K_{1} \quad$ - decreasing) if $f\left(x_{1}\right) \geq f\left(x_{2}\right)$ whenever $\quad x_{1}, x_{2} \in X$ and $x_{1} \in x_{2}+K_{1}+K($ $\left.x_{1} \in x_{2}-K_{1}-K\right)$.

Obviously, every real increasing (decreasing) function defined on any linear space ordered by an arbitrary convex cone $K$ is $\left(K+K_{1}\right)$ - increasing

( $K+K_{1}$ - decreasing), for each non - empty subset $K_{1}$ of $K$.

The next coincidence of the efficient points sets and the Choquet boundaries generalizes the main result given by Bucur, I. and Postolică, V., 1994, and can not be obtained as a consequence of the Axiomatic Potential Theory.

Theorem 5. (Postolică,V., 2008, 2009). If $A$ is any non-void, compact subset of every Hausdorff linear topological vector space $X$ and

(i) $K$ is an arbitrary, closed, convex, pointed cone in $X$;

(ii) $K_{1}$ is a non-empty subset of $K$ such that $K+K_{1}$ is closed with respect to the Hausdorff separated topology on $X$.

Then, $\operatorname{MIN}\left(A, K, K_{1}\right)\left(\operatorname{MAX}\left(A, K, K_{1}\right)\right)$ coincides with the Choquet's boundary $\partial_{S_{1}} A\left(\partial_{S_{2}} A\right)$ of $A$ with respect to the convex cone $S_{1}\left(S_{2}\right)$ of all

$K+K_{1}$ - increasing ( $K+K_{1}$ - decreasing) real continuous functions on A. Consequently, each of these sets, endowed with the corresponding trace topology, is a Baire space and a $G_{\delta}$ - subset of $X$ whenever $\left(A, \tau_{A}\right)$ is metrizable. Moreover, eff $\left.\left(A, K, K_{1}\right)=\partial_{S_{1}} A \cup \partial_{S_{2}} A\right)$.

Corollary 5.1. (i) If for every $f \in C(A)$ one denotes $\bar{f}(a)=\sup \left\{f\left(a^{\prime}\right): \mathrm{a}^{\prime} \in A \cap a+K+K_{1}\right\}$ and $\tilde{f}(a)=\sup \left\{f\left(a^{\prime \prime}\right): \mathrm{a}^{\prime} \in \mathrm{A} \cap\left(a-K-K_{1}\right\}\right.$, then

$\operatorname{eff}\left(A, K, K_{1}\right)=\bigcup_{a \in A}\{a \in A: f(a)=\bar{f}(a)(\tilde{f}(a)), \forall f \in C(A)\} ;$

(ii) $\operatorname{MIN}\left(A, K, K_{1}\right)\left(\operatorname{MAX}\left(A, K, K_{1}\right), \operatorname{MIN}\left(A, K, K_{1}\right)\left(\left(\operatorname{MAX}\left(A, K, K_{1}\right)\right) \bigcap\{a \in A: s(a) \leq 0\}\left(s \in S_{1}\right)\right.\right.$ are compact sets with respect to Choquet's topology and as usual compact subsets of $A$. 
Remark 12. Generally, $\operatorname{MIN}\left(A, K, K_{1}\right)\left(\operatorname{MIN}\left(A, K, K_{1}\right)\right)$ coincides with the corresponding Choquet boundary of A only with respect to the convex cone of all real, continuous and $K+K_{1}$ - increasing ( $K+K_{1}$ - decreasing) functions on A. Thus, for example, if $A$ is any non - empty, compact convex subset of every Hausdorff locally convex space and

$S=\{f: A \rightarrow R / f$ is continuous and concave $\}$, then its Choquet's boundary with respect to $\mathrm{S}$ coincides with the set $\operatorname{ex}(A)$ of all extreme points $\mathrm{x}$ in $A$, that is, if $y, z \in A$ and there exists $\alpha \in(0,1)$ with $\alpha y+(1-\alpha) z=x$, then $y=z=x$. The hypothesis of concavity imposed on the functions is essential for the validity of this result. Generally, even in the finite dimensional cases, an extreme point for a compact convex set is not necessary an efficient point and conversely.

Remark 13. The largest class $\zeta$ of convex cones ensuring the existence for the efficient points in all non-empty compacts subsets of every separated topological vector space was defined by Sterna Karwat, A., 1986 as follows: if $V$ is an arbitrary Hausdorff topological vector space, a convex cone $C$ belongs to $\zeta$ iff for every closed vector subspace $L$ of $V, C \cap L$ is a vector subspace whenever its closure $\overline{C \cap L}$ is a vector subspace. As we have already specified before Theorem 5 , there exists more general conditions than compactness imposed upon a non-empty set $\mathrm{A}$ in a separated locally convex space ordered by a convex cone $\mathrm{K}$ ensuring that $\operatorname{eff}(A, K) \neq \varnothing$. Perhaps our coincidence result suggests a natural extension of the Choquet boundary at least in these cases. Anyhow, Theorem 5 represents an important link between the Vector Optimization, the Potential Theory, with their Applications and a new way for the study of the properties of the efficient points sets and the Choquet boundaries. Indeed, one of the main question in Potential Theory is to find the Choquet boundaries. This fact is relatively easy for particular cases but, in general, it is an unsolved problem. Since in a lot of cases the efficient points sets contain dense subsets which can be identified by adequate optimization methods, it is possible to determine the corresponding Choquet boundaries in all these situations. Consequently, our coincidence result has its practical consequences at first for the Axiomatic Theory of Potential and its applications. At the same time, by the above coincidence result, the Choquet boundaries offer important properties for the efficient points sets.We also note that, in general, there exist no any connection between Choquet's boundary and Choquet's Integral, probably thanks to the introductions of these concepts in different temporal moments.

The above context of research suggests immediately the following open problems.

Open Problem 7. If eff $\left(A, K, K_{1}\right) \neq \varnothing$, there exist a separated locally convex space $\widehat{X}$, a (pointed), convex cone $\widehat{K} \quad$ in $\widehat{X}, \varnothing \neq \widehat{K_{1}} \subseteq \widehat{K} \quad$ and a compact set $\widehat{A} \subseteq \widehat{X}$ such that eff $\left(A, K, K_{1}\right)=\operatorname{eff}\left(\widehat{A}, \widehat{K}, \widehat{K_{1}}\right)$ (at least eff $\left(A, K, K_{1}\right)$ to be dense in eff $\left(\widehat{A}, \widehat{K}, \widehat{K_{1}}\right)$ or

conversely)?

Open Problem 8. If $T$ is a Hilbert space, $K$ is a closed, convex, pointed cone in $T, \varnothing \neq K_{1} \subseteq K$ and $A$ is a non-empty, closed, convex subset of $T$ does eff $\left(A, K, K_{1}\right)$ preserve the property of coincidence with the corresponding Choquet's boundary as in the above theorem?

Open Problem 9. The same question for each of the following cases:

(i) $T$ is a quasi - complete locally convex space, $K$ is an Isac's cone, $\varnothing \neq K_{1} \subseteq K, A$ is a $K$ - bounded and $K$ - closed set in $T$ (Postolică, V., 1995).

(ii) $T$ is a quasi - complete locally convex space, the closure $\bar{K}$ of $K$ has the properties given in (Truong, X., D. H., 1994) and A is a $K$ - bounded and $K$ - closed subset in $T$.

Open Problem 10. Generalize the concept of Choquet's boundary to non-empty complete sets and extend to non-compact sets the established strong connection by coincidence between the general efficient points sets and Choquet's boundaries. 


\section{BEST APPROXIMATION IN LOCALLY CONVEX SPACES. EFFICIENCY BY SPLINES IN H-LOCALLY CONVEX SPACES}

We conclude this Research Report with Topics on the Best Approximation (Simultaneous and Vectorial) in Locally Convex Spaces, the Efficiency (Optimization) by Splines in H - Locally Convex Spaces and the Vectorial Optimization.

Let $X$ be a Hausdorff locally convex space with the topology induced by a family $P=\left\{p_{\alpha}: \alpha \in I\right\}$ of seminorms, $x_{0} \in X$ and $G$ a non-empty subset of $X$.

Definition 6. (Isac, G., Postolică, V.,1993) $g_{0} \in G$ is said to be a best simultaneous approximation for $x_{0}$ by the elements of $G$ with respect to the family $P$ (abbreviated $g_{0}$ is a $P$ - b.s.a. of $x_{0}$ ) when

$$
p_{\alpha}\left(x_{0}-g_{0}\right) \leq p_{\alpha}\left(x_{0}-g\right) \text { for all } g \in G \text { and } p_{\alpha} \in P \text {. }
$$

If, in addition, each element $x \in X$ possesses at least one $P$ - b.s.a. in $\mathrm{G}$, then the set $G$ is called $P$ - simultaneous proximinal.

Definition 7. (Isac, G., Postolică, V., 1993) $g_{0} \in G$ is said to be a best vectorial approximation of $x_{0}$ by $G$ with respect to $P$ (abbreviated $g_{0}$ is a

$$
\left.P \text { - b.v.a. of } x_{0}\right) \text { if }\left(p_{\alpha}\left(x_{0}-g_{0}\right)\right) \in \operatorname{MIN}\left(\left\{\left(p_{\alpha}\left(x_{0}-g\right)\right): g \in G\right\}, K\right) \text { where } K=R_{+}^{I} \text {. }
$$

If, in addition, each element $x \in X$ possesses at least one $P$ - b.v.a. in $G$, then the set $G$ is called $P$ - vectorial proximinal. Several original results and properties concerning these notions were given in our above mentioned book. Concerning the $H$-locally convex spaces, it is known that this concept was introduced and studied for the first time by Precupanu, T. (1969) and defined as any Hausdorff locally convex space with the seminorms satisfying the parallelogram law. At the same time, we introduced the notion of spline function in $H$ - locally convex space (Postolică, V., 1981) and we established the basic properties of approximation and optimal interpolation for these splines. Our splines are natural extensions in $H$ - locally convex spaces of the usual abstract splines which appear in any Hilbert space like the minimizing elements for a seminorm subject to the restrictions given by a set of linear continuous functionals.

So, let now $\left(X, P=\left\{p_{\alpha}: \alpha \in I\right\}\right)$ be a $H$ - locally convex space with each seminorm $p_{\alpha}$ being induced by a scalar semiproduct (..,.) $)_{a}(\alpha \in I)$ and $M$ a closed linear subspace of $X$ for which there exist a $H$ - locally convex space $\left(Y, Q=\left\{q_{\alpha}: \alpha \in I\right\}\right)$ with each seminorm $q_{\alpha} \in Q$ generated by a scalar semiproduct $<., .{ }_{a}(\alpha \in I)$ and a linear (continuous) operator $U: X \rightarrow Y$ such that $M=$ $\left\{x \in X:(x, y)_{a}=\langle U x, U y\rangle{ }_{a}, \forall \alpha \in I\right\}$.

The space of spline functions with respect to $U$ was defined by

Postolică, V.(1981) as the U-orthogonal of $M$, that is,

$\mathbf{M}^{\perp}=\left\{x \in X:\langle U x, U z\rangle{ }_{a}=0, \forall z \in M, a \in I\right\}$. Clearly, $\mathbf{M}^{\perp}$ is the orthogonal of $M$ in the $H$ - locally convex sense, namely $(\mathrm{x}, \mathrm{y}) \forall x \in M, y \in M^{\perp}, \in I$.

Let us consider the direct sum $X^{\prime}=M \oplus \mathrm{M}^{\perp}$ and for every $x \in X^{\prime}$, we denote its projection onto $\mathrm{M}^{\perp}$ by $s_{x}$. Then, taking into account the Theorem 4 obtained by Postolică, $V$. in 1981, it follows that this spline is a best simultaneous $U$ - approximation of $x$ with respect to $\mathrm{M}^{\perp}$ since it satisfies all the next condition : $p_{\alpha}\left(x-s_{x}\right) \leq p_{\alpha}\left(x_{0}-y\right), \forall y \in \mathrm{M}^{\perp}, p_{\alpha} \in P$. 
Moreover, following the definition of the approximate efficiency, the results given in Chapter 3 of (Isac, G., Postolică, V., 1993) and the conclusions obtained by (Postolică, V., 1981,1993, 1998), we have

\section{Theorem 6.}

(i) For every $x \in X^{\prime}$ the only elements of best simultaneous and vectorial approximation with respect to any family of seminorms which generates the H-locally convex topology on $X$ by the linear subspace of splines are the spline functions $\mathrm{s}_{\mathrm{x}}$. Moreover, if $M$ and $\mathrm{M}^{\perp}$ supply an orthogonal decomposition for $X$, that is, $X=M \oplus \mathrm{M}^{\perp}$, then $\mathrm{M}^{\perp}$ is simultaneous and vectorial proximinal;

(ii) If $K=R_{+}^{I}$, then for each $\mathrm{s} \in \mathrm{M}^{\perp}$, every $\sigma \in \mathrm{M}^{\perp}$ is the only one solution of the following optimization problem:

$$
\operatorname{MIN}\left(\left\{\left(q_{\alpha}(U(\eta-s))\right): \eta \in X^{\prime}, \eta-\sigma \in M\right\}, K\right)
$$

(iii) for every $x \in X^{\prime}$ its spline function $\mathrm{s}_{\mathrm{x}}$ is the only solution for each of the next vectorial optimization problems:

$$
\begin{aligned}
& \operatorname{MIN}\left(\left\{\left(q_{\alpha}(U(s-x))\right): s \in \mathrm{M}^{\perp}\right\}, K\right), \operatorname{MIN}\left(\left\{\left(p_{\alpha}(x-y)\right): y \in \mathrm{M}^{\perp}\right\}, K\right), \\
& \operatorname{MIN}\left(\left\{\left(q_{\alpha}(U y)\right): y-x \in M\right\}, K\right) .
\end{aligned}
$$

Finally, let us consider two numerical examples in which, following

Postolică,V.,(1981), Isac, G., Postolică, V.,(1993) and Postolică, V., (1998), we specify the expressions of splines and $M$ and $\mathrm{M}^{\perp}$ realize orthogonal decompositions.

Example 5.2. Let $X=H^{m}(R)=\left\{f \in C^{m-1}(R): f^{(m-1)}\right.$ is locally absolutely continuous and $\left.f^{(m)} \in L_{\text {loc }}^{2}(R)\right\}$, $m \geq 1$ endowed with the $H$ - locally convex topology generated by the scalar semiproducts

$$
(x, y)_{k}=\sum_{h=0}^{m-1}\left[x^{(h)}(k) y^{(h)}(k)+x^{(h)}(-k) y^{(h)}(-k)\right]+\int_{-k}^{k} x^{(m)}(t) y^{(m)}(t) d t, k=0,1,2, \ldots \text { and }
$$

$Y=L_{l o c}^{2}(R)$ with the H-locally convex topology induced by the scalar semiproducts $\langle x, y\rangle_{k}=\int_{-k}^{k} x(t)$ $y(t) d t, k=0,1,2, \ldots$

If $U: X \rightarrow Y$ is the derivation operator of order $m$, then

$M=\left\{x \in H^{m}(R): x^{(h)}(n)=0, \forall h=\overline{0, \mathrm{~m}-1}, n \in Z\right\}$

and $\mathrm{M}^{\perp}=\left\{s \in H^{m}(R): \int_{-k}^{k} s^{(m)}(t) x^{(m)}(t) d t, \forall x \in M, k=0,1,2, \ldots\right\}$

We proved in (Postolică, V., 1981) that

$\mathrm{M}^{\perp}=\left\{s \in H^{m}(R): s_{/(n, n+1)}\right.$ is a polynomial function of degree $2 m-1$ at most $\}$ and if $y=\left(y_{n}\right), y^{\prime}=\left(y_{n}^{\prime}\right)$, $y^{\prime \prime}=\left(y_{n}^{\prime \prime}\right), y^{(m-1)}=\left(y_{n}^{(m-1)}\right)$ are $m$ sequences of real numbers, then there exists an unique spline $S \in \mathrm{M}^{\perp}$ satisfying the following conditions of interpolation: $S^{(h)}(n)=y^{(h)}(n)$ whenever $h=\overline{0, m-1}$ and $n \in Z$. Moreover, we observed in the paragraph 3 of (Isac, G., Postolică, V., 1993) that any spline function $S$ such as this is defined by 


$$
S(x)=p(x)+\sum_{h=0}^{m-1} c_{1}{ }^{(h)}(x-1)_{+}{ }^{2 m-1}+\sum_{h=0}^{m-1} c_{2}{ }^{(h)}(x-2)_{+}{ }^{2 m-1}+\ldots+\sum_{h=0}^{m-1} c_{0}{ }^{(h)}(-x)_{+}{ }^{2 m-1}+\ldots
$$

where $u_{+}=(|u|+u) / 2$ for every real number $u, p$ is a polynomial function of degree $2 m-1$ at most perfectly determined by the conditions $p^{(h)}(0)=y_{0}{ }^{(h)}$ and $p^{(h)}(1)=y_{1}{ }^{(h)}$ for all $h=\overline{0, \mathrm{~m}-1}$ and the coefficients $c_{n}^{(h)}(h=\overline{0, \mathrm{~m}-1}, n \in Z)$ are successively given by the general interpolation.

Therefore, for every function $f \in H^{m}(R)$, there exists an unique function denoted by $S_{f} \in \mathrm{M}^{\perp}$ such that $S_{f}^{(h)}(n)=f^{(h)}(n), \forall h=\overline{0, \mathrm{~m}-1}$ and $n \in Z$. Hence, in this case, $M$ and $\mathrm{M}^{\perp}$ give an orthogonal decomposition for the space $H^{m}(R)$.

Example 2. Let $X=F_{m}=\left\{f \in C^{m-1}(R): f^{(m-1)}\right.$ is locally absolutely continuous and $\left.f^{(m)} \in L^{2}(R)\right\}$ endowed with the $H$ - locally convex topology induced by the scalar semiproducts

$(x, y)_{n}=x(n) y(n)+\int_{R} x^{(m)}(t) y^{(m)}(t) d t, n \in Z, Y=L^{2}(R)$ with the topology generated by the inner product $(x, y)_{n}=\int_{R} x(t) y(t) d t, n \in Z$ and let $U: X \rightarrow Y$ be the derivation operator of order $m$. Then, $M=\{x \in$ $F_{m}: x(n)=0$ for all $\left.n \in Z\right\}$ and

$$
\mathbf{M}^{\perp}=\left\{s \in F_{m}: \int_{R} x^{(m)}(t) y^{(m)}(t) d t=0 \text { for every } x \in M\right\} .
$$

In a similar manner as in Example1 it may be proved that $\mathrm{M}^{\perp}$ coincides with the class of all piecewise polynomial functions of order $2 m$

(degree 2m-1 at most) having their knots at the integer points. Moreover, for every function $f$ in $F_{m}$ there exists an unique spline function $S_{f} \in \mathrm{M}^{\perp}$ which interpolates $f$ on the set $Z$ of all integer numbers, that is, $S_{f}$ satisfies the equalities $S_{f}(n)=f(n)$ for every $n \in Z$, being defined by

$S_{f}(x)=p(x)+a_{1}(x-1)_{+}{ }^{2 m-1}+a_{2}(x-2)_{+}{ }^{2 m-1}+\ldots+a_{0}(-x)_{+}{ }^{2 m-1}+a_{-1}(-x-1)_{+}{ }_{+}^{2 m-1}+\ldots$

where $u_{+}$has the same signification as in Examplel, the coefficients $a_{n}(n \in Z)$ are successively and completely determined by the interpolation conditions $S_{f}(n)=f(n), n \in \mathbb{Z}\{0,1\}$ and $p$ is a polynomial function satisfying the conditions $p(0)=f(0)$ and $p(1)=f(1)$. The uniqueness of $S_{f}$ is ensured in Theorem 2 given by (Postolică, V., 1981).

Thus, $M$ and $\mathrm{M}^{\perp}$ give an orthogonal decomposition of the space $F_{m}$ and, as in the preceding example, $\mathbf{M}^{\perp}$ is simultaneous and vectorial proximinal with respect to the family of seminorms generated by the above scalar semiproducts.

Remark 14. Our examples show that the abstract construction of splines can be used to solve also several frequent problems of optimal interpolation and approximation, having the possibility to choose the spaces and the scalar semiproducts. It is obvious that for a given (closed) linear subspace of a $H$ - locally convex space $X$, such a $H$ - locally convex space $Y$ (respectively, a linear (continuous) operator $U: X \rightarrow Y$ ) would not exist. Otherwise, the problem of best vectorial approximation by the corresponding orthogonal space of any (closed) linear subspace $M$ for the elements in the direct sum $M \oplus \mathrm{M}^{\perp}$ might be always reduced to the best simultaneous approximation. But, in general, such a possibility doesn't exist. Even in a $H$ - locally convex space it is possible that there exist best vectorial approximations and the set of all best simultaneous approximations to be empty for some element of the space. We confine ourselves to mention the following simple example. 
Example 3. Let $X=R^{N}$ endowed with the topology generated by the family $P=\left\{p_{i}: i \in N\right\}$ of seminorms defined by $p_{i}(x)=\left|x_{i}\right|(i \in N)$ for every

$x=\left(x_{i}\right) \in X$ and for each $c \geq 0$ let $G_{c}=\left\{\left(x_{i}\right) \in X: x_{i} \geq 0, \forall i \in N \& \sum_{i \in N} x_{i}=c\right\}$.

$X$ is a $P$ - simultaneous strictly convex (Isac, G., Postolică, V., 1993)

$\mathrm{H}$ - locally convex space. Nevertheless, every element of $G_{c}$ is a $P$ - b.v.a. for the origin while its

corresponding set of the best simultaneous approximations with respect to $P$ is empty.

\section{Projections in Vectorial OPTIMIZATION}

Multi - Objective Programming is an important component of the General Optimization in the Mathematical Abstract Spaces and a part of it is represented by the Pareto Optimality as the first illustrative finite dimensional example of the Economical Efficiency. Not even for the market economies there exists no an universal mathematical model. Pareto efficiency or Pareto optimality (Pareto, Vilfredo, 1909) was a central theory in economics with broad applications in game theory, engineering and the social sciences. It represents the actual finite dimensional part of the Multiobjective Programming in Vector Optimization. Thus, whenever a feasible deviation from a genuine solution $\mathrm{S}$ of an arbitrary multiobjective programme generates the improvement of at least one of the objectives while some other objectives degrade, any such a solution $\mathrm{S}$ as this is called efficient or nondominated. A system in economics and in politics is called Pareto optimal or efficient in the language of the general efficiency whenever "no individual can be made better off without another being made worse off" that is, a social state is economically efficient, or Pareto optimal, provided that "no person in society can become better off without anyone else becoming worse off". This characteristic of Pareto type efficiency has been pointed out in (Arrow, K. J., 1963, Arrow, K. J., Hahn, F. M., 1971,Aubin, J. P., 1993, Debreu Gerard, 1959, Hansson, S. O., 2004, Pareto, Vilfredo, 1909, Stewart, D. J., 2004 and the others). In terms of the alternative allocations this means that given a set of alternative allocations and a set of individuals, any movement from one alternative allocation to another that can make at least one individual better off, without making any other individual worse off is called a Pareto improvement or a Pareto optimization. An allocation of resources is named Pareto optimal whenever no further Pareto improvements can be made. If the allocation is strictly preferred by one person and no other allocation would be as good for everyone, then it is called strongly Pareto optimal. A weakly Pareto optimal allocation is one where any feasible reallocation would be strictly preferred by all agents (Zimmermann, H., 2000). Consequently, Pareto type efficiency is an important approximate criterion for evaluating the economic systems and the political policies, with minimum assumptions on the interpersonal comparability. We said "approximate criterion" because it asks "the ideal" which may not reflect, for example, the workings of real economics, thanks to the folowing restrictive assumptions necessary for the existence of Pareto efficient outcomes: the markets exist for all possible goods, being perfectly competitive and the transaction costs are negligible. In the political policies, not every Pareto efficient outcome is regarded as "desirable" (see, for instance, the strategies based on the unilateral benefits). For these reasons, Pareto optimality was accepted with some or much uncertainty and controversy, but, by Arrow's renowned impossibility theorem given in 1951 according to which "no social preference ordering based on individual orderings only could satisfy a small set of very reasonable conditions"the Pareto criterion being one of them, it remains "plausible and uncontroversial" (Rosencrantz, Holger, 2005). Usually, the concept of efficiency replaces the notion of optimality in multiple criteria optimization because whenever the solutions of a multiple - objectives program exist in Pareto'sense, they cannot be improved following the ordering induced by the cone. Seemingly, the concept of efficiency is equivalent to the optimality but, in reality, the optimality represents a particular case of the efficiency, that is, "the best approximation" of all the efficient points. The next section is dedicated to the main kind of optimization problems in $R^{n}$. We also notice that the finite dimensionality of the background space is essential in contrast with the general ordered vector spaces, since it controls the results at least thanks to the approximate numerical methods for the efficient points. 


\section{Multi - Objective Optimization Programs}

Let $E$ be an arbitrary non - empty set and let $f=\left(f_{1}, f_{2}, \ldots, f_{n}\right): E \rightarrow R^{n}\left(n \in N^{*}, n \geq 2\right)$ a vector - valued function. The main general strong $\left(P_{1}\right),\left(P_{2}\right)$ and vectorial $\left(P_{3}\right),\left(P_{4}\right)$ respectively, Optimization Problems that can be formulated in this context are the following:

$$
\left(P_{1}\right) \operatorname{SMIN}\{f(x): x \in E\}
$$

where $x_{0} \in E$ is a strong minimum solution if $f\left(x_{0}\right) \leq f(x), \forall x \in E$;

$$
\left(P_{2}\right) \operatorname{SMAX}\{f(x): x \in E\}
$$

with $y_{0} \in E$ strong maximum solution if $f\left(y_{0}\right) \geq f(x), \forall x \in E$;

$$
\begin{aligned}
& \left(P_{3}\right) \operatorname{VMIN}\{f(x): x \in E\} \\
& \left(P_{4}\right) \operatorname{VMAX}\{f(x): x \in E\}
\end{aligned}
$$

In order to define the vectorial solutions, we need to know the possibility of "comparison" in $R^{n}$. Let $R_{+}^{n}=\left\{\left(x_{i}\right) \in R^{n}: x_{i} \geq 0, \forall i=\overline{1, n}\right\}$ be the usual ordering cone. If $a=\left(a_{i}\right), b=\left(b_{i}\right) \in R^{n}$, then it is possible to have:

1) $a \leq b \Leftrightarrow a_{i} \leq b_{i}, \forall i=\overline{1, n} \Leftrightarrow b-a \in R_{+}^{n}$;

2) $a<b \Leftrightarrow a_{i} \leq b_{i}, \forall i=\overline{1, n}$ with at least one of these inequalities being strict, that is

$$
b-a \in R_{+}^{n} \backslash\{\theta\}, \text { where } \theta=(0,0, \ldots, 0) \in R_{+}^{n} ;
$$

3)

$$
\begin{aligned}
& a \ll b \Leftrightarrow a_{i}<b_{i}, \forall i=\overline{1, n} \Leftrightarrow b-a \in \operatorname{int}\left(R_{+}^{n}\right)= \\
& =\left\{x_{0} \in R_{+}^{n}: \exists B\left(x_{0}, \varepsilon>0\right)=\left\{x \in R^{n}:\left\|x-x_{0}\right\|<\varepsilon\right\} \subset R_{+}^{n}\right\},
\end{aligned}
$$

where $\|\|:. R^{n} \rightarrow R_{+}$is the usual norm in $R^{n}$ given by $\|u\|=\sqrt{\sum_{i=1}^{n}\left|u_{i}\right|^{2}}, \forall u=\left(u_{i}\right) \in R^{n}$

Thus, we are led to say that $x_{0}$ is a vectorial minimum solution of $\left(P_{3}\right)$ if there exists no $x \in E$ such that $f(x)<f\left(x_{0}\right)$, which is equivalent with each of the next properties:

(i) $f(E) \cap\left[f\left(x_{0}\right)-R_{+}^{n}\right]=\left\{f\left(x_{0}\right\}\right.$;

(ii) $f(E) \bigcap\left[f\left(x_{0}\right)-R_{+}^{n} \backslash\{\theta\}\right]=\varnothing$;

(iii) $R_{+}^{n} \cap\left[f\left(x_{0}\right)-f(E)\right]=\{\theta\}$;

(iv) $\left(R_{+}^{n} \backslash\{\theta\}\right] \cap\left[f\left(x_{0}\right)-f(E)\right]=\varnothing$; 
(v) $\left[f(E)+R_{+}^{n}\right] \cap\left[f\left(x_{0}\right)-R_{+}^{n} \backslash\{\theta\}\right]=\varnothing$.

These relations show that $f\left(x_{0}\right)$ is a fixed point for each of the following multifunctions:

$$
\begin{gathered}
F_{1}: f(E) \rightarrow f(E), F_{1}(t)=\left\{\alpha \in f(E): f(E) \cap\left(\alpha-R_{+}^{n}\right) \subseteq t+R_{+}^{n}\right\}, \\
F_{2}: f(E) \rightarrow f(E), F_{2}(t)=\left\{\alpha \in f(E): f(E) \cap\left(t-R_{+}^{n}\right) \subseteq \alpha+R_{+}^{n}\right\}, \\
F_{3}: f(E) \rightarrow f(E), F_{3}(t)=\left\{\alpha \in f(E):\left(f(E)+R_{+}^{n}\right) \cap\left(\alpha-R_{+}^{n}\right) \subseteq t+R_{+}^{n}\right\}, \\
F_{4}: f(E) \rightarrow f(E), F_{4}(t)=\left\{\alpha \in f(E):\left(f(E)+R_{+}^{n}\right) \cap\left(t-R_{+}^{n}\right) \subseteq \alpha+R_{+}^{n}\right\},
\end{gathered}
$$

that is, $f\left(x_{0}\right) \in F_{i}\left[f\left(x_{0}\right)\right]$ for every $i=\overline{1,4}$. By replacing $R_{+}^{n}$ with $\left(-R_{+}^{n}\right)$, one obtains the concept of Vectorial Maximum Solution for $\left(P_{4}\right)$. Clearly, any vector optimization program (which has its origin in the usual ordered Euclidean spaces programs thanks to the Pareto type Optimality of the vector valued real functions) includes the corresponding Strong Optimization Programme. Moreover, it is possible to replace the usual convex cone $R_{+}^{n}$ by any other convenient convex cone in $R^{n}$. In this way, we are sent to examine for a set $\varnothing \neq A \subseteq R^{n}$ the following subsets

a) $\operatorname{SMIN}(A)=\left\{a_{0} \in A: a-a_{0} \in R_{+}^{n}, \forall a \in A\right\}$ (the set of all strong minimum points);

$\beta) \operatorname{SMAX}(A)=\left\{\overline{a_{0}} \in A: \overline{a_{0}}-a \in R_{+}^{n}, \forall a \in A\right\}$ (the set of all strong maximum points);

$\gamma) \operatorname{VMIN}(A)=\left\{b_{0} \in A: A \cap\left(b_{0}-R_{+}^{n}\right)=\left\{b_{0}\right\}\right\}$ (the set of all vectorial minimum points);

d) $\operatorname{VMAX}(A)=\left\{\overline{b_{0}} \in A: A \cap\left(\overline{b_{0}}+R_{+}^{n}\right)=\left\{\overline{b_{0}}\right\}\right\}$ (the set of all vectorial maximum points).

All the Strong Optimal Points for $A$ are contained in the set $S(A)=\operatorname{SMIN}(A) \cup \operatorname{SMAX}(A)$ while the set of all Efficient points (Vectorial or Strong) is defined by

$V(A)=\operatorname{VMIN}(A) \cup \operatorname{VMAX}(A)=\operatorname{eff}\left(A, R_{+}^{n}\right)$, the last equality being in accordance with (Postolică, V., 2017). Moreover, $a_{0} \in e f f\left(A, R_{+}^{n}\right)$ if and only if it is a critical (ideal or balance) point for the generalized dynamical systems $\Gamma_{\mp}: A \rightarrow 2^{A}$ defined by $\Gamma_{\mp}(a)=A \cap\left(a \mp R_{+}^{n}\right), \forall a \in A$. In this way, eff $\left(A, R_{+}^{n}\right)$ describes the balance extremum moments for $\Gamma_{\mp}$, which in the natural market context, expresses the competitive equilibrium/non - equilibrium consisting of the general relation price/consumption. The first main connection between the above mentioned sets is the following, established in the general conditions offered by any real or complex vector space ordered by an arbitrary convex cone. Following Theorem 1, if $S(A) \neq \varnothing$, then $V(A)=S(A)$. In fact, $\operatorname{SMIN}(A) \neq \varnothing(\operatorname{SMAX}(A) \neq \varnothing) \Rightarrow \operatorname{VMIN}(A)=\operatorname{SMIN}(A)(\operatorname{VMAX}(A)=\operatorname{SMAX}(A))$.

Open Problem 11. Characterizations of the equivalence

$$
S(A)=\varnothing \Leftrightarrow V(A)=A .
$$

As we mentioned in Remark 4, this result is also important for for the numerical methods since it says that, if there exist"strong optimal points", then these elements are the only "vectorial optimal points". 
Remark 15. In our opinion, following the most general context of the ordered linear spaces, any optimization programme can be generally formulated as follows: let $E$ be a non-empty set, let $X$ be a vector space ordered by a convex cone $K$ and let $f: E \rightarrow(X, K)$ be an objective function. The problem consists to look for the optimums of the set $E$ following the properties of its image $f(E)=\{f(x): x \in E\}$ in $X$ by $K$ and conversely.

\section{FUtURE RESEARCH DiRECTIONS}

This research work can also be considered as a study in which the approximate solutions including those usual to vector optimization programs in ordered (topological) vector spaces are investigated. The listed properties of these solutions allow developments. Several new facts about the relationships between the approximate efficiency and the scalarized problems can be formulated. As we have specified, the family of Isac's cones represent the largest class of ordering cones in Hausdorff locally convex spaces ensuring the existence and the adequate properties of the general efficient points sets involved in optimization, following different completeness types instead of compactness. Consequently, one of the main goal of the next research is to identify new applications of Isac's cones and new links with other important classes of convex cones in separated locally convex spaces.

\section{ACKNOWLEDGEMENTS}

The author would like to express his gratitude to the colleagues from the International Journal of Scientific and Innovative Mathematical Research.

\section{REFERENCES}

[1] AGARWAL, R.P., O'REGAN - A Note on Equilibria for Abstract Economies. Math. Computer Modelling, 34(2001), $331-343$.

[2] AUBIN, J.P. - Optima and Equilibria. An Introduction to Nonlinear Analysis. Springer - Verlag Berlin Heidelberg, 1993.

[3] BAHYA, A. O. - Ensembles Coniquement Bornés et Cônes Nucléaires dans les Éspaces Localement Convexes Separés. Théses de Doctorat de 3 - ème Cycle en Mathématiques. L'école Normale Superieure Takaddoum, Rabat (Maroc), 1989.

[4] BENSON, H.P. - The Vector Maximization Problem: Proper Efficiency and Stability. SIAM J. Appl. Math. 32, 1977, 64 - 72.

[5] BENSON, H.P. - An Improved Definition of Proper Efficiency for Vector Minimization with respect to Cones. J. Math. Anal. Appl. 79, 1979, 232 - 241.

[6] BENSON, H.P. - Efficiency and Proper Efficiency for Vector Minimization with respect to Cones. J. Math. Anal. Appl. 93, 1983, 273 - 289.

[7] BOBOC, N., BUCUR, GH. - Convex Cones of Continuous Functions on Compact Spaces (in Romanian). Publishing House of Romanian Academy, Bucharest, 1976.

[8] BORWEIN, J.M. - Proper Efficient Points for Maximization with respect to Cones. SIAM J. Control Optim., 15, 1977, $57-63$.

[9] BORWEIN, J.M. - On the Existence of Pareto Efficient Points. Math Oper. Res. 8, no. 1, 1983, 64 - 73.

[10] BUCUR, I., POSTOLICĂ, V. - A Coincidence Result between Sets of Efficient points and Choquet Boundaries in Separated Locally Convex Spaces. Research Report at the $4^{\text {th }}$ Workshop of the German Working Group on Decision Theory, Hotel Talblick, Holzhau, Germany, March 14 - 18, 1994. Published in Optimization, 36, 1996, 231 - 234.

[11] CARDINALI, T. and PAPALINI, F. - Fixed point Theorems for Multifunctions in Topological Vector Spaces. J. Math. Anal. Appl., 186, 1994,769 - 777.

[12] DAUER, J. P., GALLAGHER, R. J. - Positive Proper Efficient points and related Cone Results in Vector Optimization Theory. SIAM J. Control Optim. 28(1), 1990, 158 - 172.

[13] DOMINIAK CEZARY - Multicriteria Decision Aid under Uncertainty. In: Multiple Criteria Decision Making'05 (Ed.Tadeusz Trzaskalik), Publisher of The Karol Adamiecki University of Economics in Katowice, 2006, 63 - 81.

[14] EHRGOTT, MATTHIAS - Multicriteria Optimization. Springer Berlin - Heidelberg, 2005.

[15] FISHBURN, P. C. - Foundations of Risk Management Science I. Risk or Probable Loss. Management Science, 30, 1984, $396-406$. 
[16] GASS SAUL, I., HARRIS CARL, M. - Enciclopedia of Operations Research and Management Science. Centennial Edition. Kluwer Academic Publishers, Boston, Dordrecht, London, 2001.

[17] GEOFFRION, A. M. - Proper Efficiency and the Theory of Vector Maximzation. J. Math. Anal. Appl., 22, 1968, $618-630$.

[18] HARTLEY, R. - On Cone- Efficiency, Cone Convexity and Cone - Compactness. SIAM J. Appl. Math., 34 (2), 1978, 211- 222.

[19] HENING, M. I. - Proper Efficiency with respect to Cones. J. Optim. Theory. Appl., 36,1982, 387 - 407.

[20] HEYNE, PAUL - Efficiency. Research Report, University of Washington, 2000.

[21] HYERS, D. H., ISAC, G., RASSIAS, T. M. - Topics in Nonlinear Analysis and Applications. World Scientific Publishing Co, Pte. Ltd., 1997.

[22] KIM, W. K., TAN, K.K. - New Existence Theorems of Equilibria and Applications. Nonlinear Anal., 47(2001) $531-542$.

[23] ISAC, G. - Cônes Localement Bornés et Cones Completement Reguliers. Applications a l'Analyse Non Linéaire Moderne. Université de Sherbrooke, no. 17, 1980.

[24] ISAC, G. - Points Critiques des Systémes Dinamiques. Cônes Nucléaires et Optimum de Pareto, Research Report, Royal Military College of St. Jean, Québec, Canada, 1981.

[25] ISAC, G. - Sur l'Existence de l'Optimum de Pareto, Riv. Mat. Univ. Parma, 4(9), 1983, 303 - 325.

[26] ISAC, G. - Supernormal Cones and Absolute Summability, Libertas Mathematica, 5, 1985, 17 - 32.

[27] ISAC, G., POSTOLICĂ, V. - The Best Approximation and Optimization in Locally Convex Spaces, Verlag Peter Lang GmbH, Frankfurt am Main, Germany, 1993.

[28] ISAC, G. - Pareto Optimization in Infinite Dimensional Spaces: the Importance of Nuclear Cones, J. Math. Anal. Appl., 182( 2), 1994, 393 - 404.

[29] ISAC, G. - On Pareto Efficiency. A General Constructive Existence Principle. Research Report (16 pp.), Department of Mathematics and Computer Science, Royal Military College of Canada, 1998.

[30] ISAC, G., BAHYA, A., O. - Full Nuclear Cones associated to a Normal Cone. Application to Pareto Efficiency. Applied Mathematics Letters, 15, 2002, 633 - 639.

[31] ISAC, G., BULAVSKY, V. A., KALASHNIKOV, V. V. - Complementarity, Equilibrium, Efficiency and Economics. Kluwer Academic Publishers, 2002.

[32] ISAC, G. - Nuclear Cones in Product Spaces. Pareto Efficiency and Ekeland type Variational Principles in Locally Convex Spaces (Preprint, Royal Military College of Canada, 2003).

[33] ISAC, G., TAMMER, CHR. - Nuclear and Full Nuclear Cones in Product Spaces. Pareto Efficiency and Ekeland type Variational Principles (Preprint, Royal Military College of Canada and University of Hall, 2003).

[34] ISAC, G., POSTOLICĂ, V. - Full Nuclear Cones and a relation between Strong Optimization and Pareto Efficiency. J. Global Optimiz., 32, 2005, 507 - 516.

[35] JABLONSKY, J.- A Slack based Model for Measuring Supe - Efficiency in Data Envelopment Analysis. In: Multiple Criteria Decision Making'05 (Ed.Tadeusz Trzaskalik), Publisher of The Karol Adamiecki University of Economics in Katowice, 2006, 101-112.

[36] KRAMAR, E. - Locally Convex Topological Vector Spaces with Hilbertian seminorms. Rev. Roum. Math Pures et Appl., Tome XXVI, no. 1, 1981, 55 - 62.

[37] KRASNOSELSKI, M. A. - Positive Solutions of Operator Equations. Gröningen, Noordhoff,1964.

[38] LORIDAN, P. $-\varepsilon$ - Solutions of Vector Minimization Problems. J. Optim. Theory Appl., 43(2), 1984, $265-276$.

[39] LUC, D. T. - Theory of Vector Optimization. Springer-Verlag, 1989.

[40] LUPTÁČIK, M., BÖHM, B. - Measuring Eco - Efficiency an a Leontief input - output Model. In: Multiple Criteria Decision Making'05 (Ed.Tadeusz Trzaskalik), Publisher of The Karol Adamiecki University of Economics in Katowice, 2006, 121-135.

[41] NACHBIN, L. - Topology and Order. New York, Van Nostrand, 1965.

[42] NÉMETH, A. B. - Between Pareto Efficiency and Pareto $\varepsilon$-Efficiency. Optimization,10(5),1989, 615 637.

[43] NG., K. F., ZHENG, X. Y. - Existence of Efficient Points in Vector Optimization and Generalized Bishop Phelps Theorem. JOTA, 115, 2002, 29 - 47.

[44] PARETO, VILFREDO - Manuel d'Économie Politique, Girard et Brière, 1909.

[45] PARK, S. - Some Coincidence Theorems on Acyclic Multifunctions Applications.In: Tan, K.- K(Ed) Fixed Point Theory and Applications, World Sci. NJ, 1992, 248 -277. 
[46] PATRICHE, M. - Fixed Point Theorems for Nonconvex Valued Correspondences and Applications in Game Theory. Fixed point Theory, 14(2013), no. 2, 435 - 446.

[47] PERESSINI, A. L. - Ordered Topological Vector Spaces. Harper 8 Row, New York, 1967.

[48] PIETCH, A. - Nuclear Locally Convex Spaces. Springer - Verlag, 1972.

[49] POSTOLICĂ, V. - Spline Functions in H - Locally Convex Spaces. An. Şt. Univ. “Al. I. Cuza”, Iaşi, 27, $1981,333-338$.

[50] POSTOLICĂ, V. - Existence Conditions of Efficient points for Multifunctions with Values in Locally Convex Spaces. Stud. Cerc. Mat., Tom 41, no. 4, 1989, 325 - 331.

[51] POSTOLICĂ, V. - New Existence Results for Efficient points in Locally Convex Spaces ordered by Supernormal Cones, J. Global Optimiz., 3, 1993, 233 - 242.

[52] POSTOLICĂ, V. - An Extension to Sets of Supernormal Cones and Generalized Subdifferentials. Optimization, 29, 1994, 131 - 139.

[53] POSTOLICĂ, V. - Properties of Pareto Sets in Locally Convex Spaces. Optimization, 34, 1995, 223 - 229.

[54] POSTOLICĂ, V. - Recent Conditions for Pareto Efficiency in Locally Convex Spaces. Research Report at the Joint International Meeting Euro XV-INFORMS XXXIV, Barcelona, Spain, July 14 - 17, 1997.

[55] POSTOLICĂ, V. - Properties of Efficient points Sets and related Topics. Research Report at The Second International Conference on Multi - Objective Programming and Goal Programming, Torremolinos, Spain, May 16-18, 1996. Published in Advances in Multiple Objective and Goal Programming, Lecture Notes in Economics and Mathematical Systems, 455, (Eds.: Rafael Caballero, Francisco Ruiz, Ralph E. Steuer), 1997, $201-209$.

[56] POSTOLICĂ, V. - A Method which Generates Splines in H - Locally Convex Spaces and Connections with Vectorial Optimization. Positivity, 2 (4), 1998, 369 - 377.

[57] POSTOLICĂ, V. - Conditions for Pareto Efficiency in Locally Convex Spaces. Mathematical Reports of Romanian Academy 2, 1(51), April - June, 1999, 257 - 269.

[58] POSTOLICĂ, V., SCARELli, A. - Some Connections between Best Approximation, Vectorial Optimization and Multicriterial Analysis. Nonlinear Analysis Forum, 5, 2000, 111 - 123.

[59] POSTOLICĂ, V. SCARELLI, A., VENZI, L. - On the Equilibrium of a Multidimensional Ecosystem. Nonlinear Analysis Forum, 6(2), 2001, 321 - 335.

[60] POSTOLICĂ, V. - New Existence Results of Solutions for Vector Optimization Programs with Multifunctions. Optimization, vol. 50, 2001, 253 - 263.

[61] POSTOLICĂ, V. - Pareto Efficiency, Choquet Boundaries and Operators in Hausdorff Locally Convex Spaces. Nonlinear Analysis Forum, 7(2), 2002, 215 - 230.

[62] POSTOLICĂ, V. - A Class of Generalized Dynamical Systems in Connection with Pareto Efficiency and related Topics. Research Paper submited to the International Conference "Complex Systems, Intelligence and Modern Technological Applications", Cherbourg, France, September 19 - 22, 2004. Published in the coresponding Electronics Proceedings Volume.

[63] POSTOLICĂ, V. - Choquet Boundaries and Efficiency. Computer and Mathematics with Applications, 55, 2008, $381-391$.

[64] POSTOLICĂ, V. - Approximate Efficiency in Infinite Dimensional Ordered Vector Spaces. International Journal of Applied Management Science (IJAMS), Vol. 1, No. 3, 2009, 300 - 314.

[65] POSTOLICĂ, V. - Isac's Cones in General Vector Spaces. Published as Chapter 121, Category: Statistics, Probability, and Predictive Analytics, vol.3, p.1323-1342 in Encyclopedia of Business Analytics and Optimization 5 Vols. (Ed. John Wang, PhD., Montclair State University, USA,), IGI Global, ISBN: 978-14666 - 5202-6(hardcover); 978-1-4666-5203-3(ebook); 978-1-4666-5205-7 (print\&perpetual access); DOI: 10.4018/978-1-4666-5205-7; (C) 2014; 2754 pages.

[66] POSTOLICĂ, V. - Isac's Cones at Various Topologies. Int. J. Data Science, Vol. 1, No. 1, 2015, p. 73 83, DOI:10.1504/IJDS2015.069048.

[67] POSTOLICĂ, V. - Isac's Cones. British Journal of Applied Science \& Technology, 15(4):1-8, 2016; DOI:10.9734/BJAST/2016/24007.

[68] POSTOLICĂ, V. - General Efficiency. Invited Research Work at the $7^{\text {th }}$ European Congress of Mathematics, July 18 - 22, 2016, TU Berlin, Germany. Presented in the Contributed Session 16, CS-16-B: "Optimization and Control" on Tuesday, July 19, 2016, 10:40. The Abstract published in the Book of Abstracts, p. 606.

[69] PRECUPANU, T. - Espaces Linéaires à Semi - normes Hilbertiennes. An. Şt. Univ. “Al. I. Cuza”, Iaşi, $15,1969,83-93$.

[70] PRECUPANU, T. - Scalar Minimax Properties in Vectorial Optimization. International Series of Numerical Mathematics, Birkhäuser Verlag Basel, 107, 1992, 299 - 306. 
[71] SONGSAK SRIBOONCHITTA, WING-KEUNG WONG, SOMPONG DHOMPONGSA, HUNG, T. NGUYEN - Stochastic Dominance and Applications to Finance, Risk and Economics. CRC Press, Taylor and Francis Group, October 2009, 456 pp., ISBN: 978-1-4200-8266-1.

[72] SONNTAG, Z., ZĂLINESCU, C. - Comparison of Existence Results for Efficient Points. JOTA, 105, 2000, 161-188.

[73] STERNA - KARWAT, A. - On the Existence of Cone Maximal points in Real Topological Linear Spaces. Israel Journal of Mathematics, 54(1), 1986, 33 - 41.

[74] STEWART, D. J. - Uncertainties in MCDA. In: Multi Criteria Decision Analysis (Ed. P. Greco). Springer - Verlag, 2004.

[75] TREVES, F. - Locally Convex Spaces and Linear Partial Differential Equations. Springer - Verlag, New York, 1967.

[76] TRUONG, X. D. H. - A Note on a Class of Cones ensuring the Existence of Efficient points in Bounded Complete sets. Optimization, 31, 1994, 141-152.

[77] TRUONG, X. D. H. - On the Existence of Efficient points in Locally Convex Spaces. J. Global Optimiz., 4, 1994, $265-278$.

[78] WANTAO, FU, YONGHONG, CHENG - On the Super Efficiency in Locally Convex Spaces. Nonlinear Analysis 44 (2001), $821-828$.

[79] ZHANG CONG - JUN - Generalized Bi-Quasi-Variational Inequalities and Generalized QuasiVariational Inequalities. Acta Analysis Functionalis Applicata, 7(2), 2005, 116 - 122.

[80] ZIMMERMANN, H. - An Application - Oriented View of Modeling Uncertainty. European Journal of Operation Research, 122, 2000, 190 - 198.

\section{AUTHOR'S BIOGRAPHY}

Vasile Postolică received his Bachelor's degree in Mathematics from the University"Al.I.Cuza" Iaşi, Romania, Faculty of Mathematics, completed by the Doctorate degree. He is full professor, "Vasile Alecsandri" University of Bacău, Faculty of Sciences, Department of Mathematics, Informatics and Education Sciences. Titular member of: the Romanian Academy of Scientists, the Romanian Society of Mathematicians, the American Mathematical Society, the Society for Industrial and Applied Mathematics, the Association of European Operational Research Societies (EURO - EURO Working Group Multicriteria Decision Aiding (EWG - MCDA)), the International Society for Optical Engineering (U.S.A.), MCDM - Society (Univ.of Georgia, U.S.A.), the Planetary Society, Pasadena, CA, GAMM - the International Association of Applied Mathematics and Mechanics, Germany, EMS - the European Mathematical Society, Finland, the International Association of Mathematical Physics, the International Federation of Nonlinear Analysts.: www.postolica.ambra.ro.

Citation: V. Postolică, " Efficiency and Vectorial Optimization ", International Journal of Scientific and Innovative Mathematical Research, vol. 6, no. 2, p. 41-65, 2018., http://dx.doi.org/10.20431/23473142.0602004

Copyright: (C) 2018 Authors. This is an open-access article distributed under the terms of the Creative Commons Attribution License, which permits unrestricted use, distribution, and reproduction in any medium, provided the original author and source are credited. 